\title{
Jamming at zero temperature and zero applied stress: The epitome of disorder
}

\author{
Corey S. O'Hern* and Leonardo E. Silbert \\ Department of Chemistry and Biochemistry, UCLA, Los Angeles, California 90095-1569, USA \\ and James Franck Institute, The University of Chicago, Chicago, Illinois 60637, USA \\ Andrea J. Liu \\ Department of Chemistry and Biochemistry, UCLA, Los Angeles, California 90095-1569, USA \\ Sidney R. Nagel \\ James Franck Institute, The University of Chicago, Chicago, Illinois 60637, USA
}

(Received 17 April 2003; published 25 July 2003)

\begin{abstract}
We have studied how two- and three-dimensional systems made up of particles interacting with finite range, repulsive potentials jam (i.e., develop a yield stress in a disordered state) at zero temperature and zero applied stress. At low packing fractions $\phi$, the system is not jammed and each particle can move without impediment from its neighbors. For each configuration, there is a unique jamming threshold $\phi_{c}$ at which particles can no longer avoid each other, and the bulk and shear moduli simultaneously become nonzero. The distribution of $\phi_{c}$ values becomes narrower as the system size increases, so that essentially all configurations jam at the same packing fraction in the thermodynamic limit. This packing fraction corresponds to the previously measured value for random close packing. In fact, our results provide a well-defined meaning for "random close packing" in terms of the fraction of all phase space with inherent structures that jam. The jamming threshold, point $J$, occurring at zero temperature and applied stress and at the random-close-packing density, has properties reminiscent of an ordinary critical point. As point $J$ is approached from higher packing fractions, power-law scaling is found for the divergence of the first peak in the pair correlation function and in the vanishing of the pressure, shear modulus, and excess number of overlapping neighbors. Moreover, near point $J$, certain quantities no longer self-average, suggesting the existence of a length scale that diverges at $J$. However, point $J$ also differs from an ordinary critical point: the scaling exponents do not depend on dimension but do depend on the interparticle potential. Finally, as point $J$ is approached from high packing fractions, the density of vibrational states develops a large excess of low-frequency modes. Indeed, at point $J$, the density of states is a constant all the way down to zero frequency. All of these results suggest that point $J$ is a point of maximal disorder and may control behavior in its vicinity-perhaps even at the glass transition.
\end{abstract}

DOI: 10.1103/PhysRevE.68.011306

PACS number(s): 81.05.Rm, 83.80.Iz, 64.70.Pf, 82.70.-y

\section{INTRODUCTION}

The nature of the glass transition has been called probably "the deepest and most interesting unsolved problem in solidstate theory [1]." The nature of granular materials has also been said to lead to equally deep questions in statistical physics: "One might even say that the study of granular materials gives one a chance to reinvent statistical mechanics in a new context [2]." Indeed, only a few years ago, the state of understanding of granular matter was compared to "the level of solid-state physics in 1930 [3]." There is no doubt that there are hard and deep problems associated with both types of systems and it may seem, at the outset, foolish to try to study both problems simultaneously. However, there have been significant advances in both fields of study that indicate that these problems are perhaps intimately related. They both deal with amorphous systems of particles in which the dynamics is perched precariously near a transition between a flowing and a static state; that is, both systems are close to a jamming threshold where all dynamics ceases. One question

\footnotetext{
*Permanent address: Department of Mechanical Engineering,
} Yale University, New Haven, CT 06520-8284, USA. that one can ask is whether there is something generic about such transitions so that the freezing of a liquid into a glass can profitably be compared to the arrest of a flowing granular material, or a suspension, as external stresses are reduced below the yield stress. In other words, can one study systems that can explore different states either through thermal fluctuations or through externally applied stresses, and search for unifying concepts that describe their arrested dynamics as different aspects of a more general "jamming" behavior [4]?

Our approach to this problem is to describe both glassy systems and granular ones using the concept of a "jamming phase diagram." In such a diagram, the "phase boundary" marks the point where the response of the system has become so sluggish as to make it appear solid on any experimental time scale. Using this framework, one can gain insight into the relationship between athermal jamming and thermal glass transitions, and appreciate what are the control variables that govern dynamical slowing down under many different conditions. In this paper, we describe simulations of a model liquid with frictionless, finite-range repulsive interactions. Because the potentials fall to zero at some fixed finite radius, such a system may be a starting point for understanding macroscopic granular or colloidal systems as well as liquids. For such potentials, there is one special point, 
at zero temperature and zero applied shear stress on the surface separating the jammed and unjammed regions, which has exceptional and unique properties. The goal of this paper is to elucidate some of the important properties of this specific jamming transition in depth. We have found that the transition near this point has some aspects that resemble a critical point and other properties that are not expected for a normal second-order transition. However, just as with a more conventional critical point, there is the tantalizing possibility that it may control the region around it and thereby govern the nature of the entire jamming surface in the phase diagram.

We will first describe what is meant by jamming and what systems may profitably be studied under this rubric. We will then describe the jamming phase diagram and show the important consequences that can be drawn from it. The nature of the transition at zero temperature and zero applied shear stress will then be described to show why it is such an important and unique transition.

\section{A. Systems that jam}

Jamming occurs when a system develops a yield stress in a disordered state [4]. In many cases, it is difficult to tell whether a system has an infinite stress relaxation time (and hence a yield stress), or whether it has a finite stress relaxation time that exceeds the time scale of one's measurement. Therefore, an alternate definition is that jamming occurs when a system develops a stress relaxation time that exceeds a reasonable experimental time scale in a disordered state. According to these definitions, many systems jam. Granular materials can flow when they are shaken or poured through a hopper, but jam when the shaking intensity or pouring rate is lowered [5]. Colloidal suspensions of particles are fluid, but jam when the pressure or packing fraction is raised [6]. Foams and emulsions (concentrated suspensions of deformable bubbles or droplets) flow when a large shear stress is applied, but jam when the shear stress is lowered below the yield stress [7]. It should be emphasized here that granular materials, foams, and dense emulsions are athermal in the sense that ordinary room-temperature thermal fluctuations are too insignificant to allow the system to explore phase space. However, for other systems - typically those consisting of smaller particles, such as molecular liquidstemperature plays an important, if not dominant, role. These liquids jam (if crystallization does not intervene first) as temperature is lowered or density is increased-this is the glass transition [8]. There are a number of striking similarities in the phenomenology of these different transitions. Despite much effort, no significant static structural signature-as opposed to a kinetic slowing down - of jamming has been observed experimentally in any of these systems [9]. However, we have proposed that such a signature can be observed in a quantity initially measured for granular materials [10]. Another similarity among the different systems is that the increase of the stress relaxation time tends to be superArrhenius as a function of the control parameter [11]. In addition, all systems show kinetic heterogeneities near the onset of jamming, where particle mobilities become hetero-

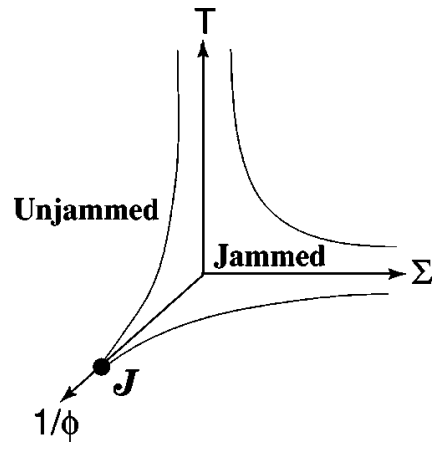

FIG. 1. "Jamming phase diagram." The jammed region, near the origin, is enclosed by the depicted surface. The point labeled $J$ is the boundary of the jammed region at $T=0$ and $\Sigma=0$. Adapted from Ref. [13].

geneous in space and intermittent in time [12]. However, the parameters that control jamming (temperature for the glass transition, applied shear stress for a foam, packing fraction for a colloidal suspension) are so different that previously it was difficult to see how to compare the jamming transitions at a quantitative level.

\section{B. Jamming phase diagram}

We proposed in Ref. [13] that different routes to kinetic arrest can be tied together by a "jamming phase diagram," shown schematically in Fig. 1. The shape of the jamming surface may be different for different systems. The choice of axes is dictated by the parameters that control the transition to jamming in different systems, namely, temperature $T$, density or packing fraction $\phi$, and shear stress $\Sigma$. Note that $T$ and $\phi$ are traditional axes for phase diagrams, but $\Sigma$ is not. In the unjammed regime, the system flows at nonzero $\Sigma$, so $\Sigma$ is a nonequilibrium axis. Why should there be such an axis in the jamming phase diagram? One reason is that shear stress introduces fluctuations in the unjammed regime by forcing the system to explore different packing configurations. Recent studies show that such fluctuations can be described by an "effective temperature" that has many of the attributes of a true temperature [14-17]. Moreover, the dynamics of a sheared system whose effective temperature is lowered toward jamming are quantitatively similar to the dynamics of an equilibrium system whose temperature is lowered toward the glass transition $[18,19]$. These results help to justify the existence of shear stress as an axis on the phase diagram.

The ordinary phase diagram for the glass transition lies in the vertical plane coming out of the page of Fig. 1, namely, the $(1 / \phi)-T$ plane. At high packing fraction, there is a transition between a supercooled liquid and a glass that occurs at $T_{g}$. (Although the relaxation times appear as if they will diverge close to the transition line, it is impossible in practice to track their increase past the times scales accessible to experiment. Thus, the transition line $T_{g}$ marks the position where the relaxation time has reached some large threshold. Its exact position may depend to a small extent on the largest time that an experimentalist is willing to run an experiment. This definition corresponds to the conventional one used for 
$T_{g}$ in glass-forming liquids.) As the packing fraction is lowered, $T_{g}$ normally decreases [20]. This glass-transition line is represented by the curve separating the jammed (i.e., glass) and unjammed (i.e., liquid) regions in the $(1 / \phi)-T$ plane. The ordinary phase diagram for a foam or emulsion would be in the horizontal plane coming out of the page, namely, the $(1 / \phi)-\Sigma$ plane of Fig. 1. At a fixed packing fraction, one must apply a shear stress higher than the yield stress in order for the system to flow at an experimentally measurable shear rate. Thus, the yield stress as a function of packing fraction is the curve that separates the jammed and unjammed regions in this plane. As the packing fraction decreases toward close packing, the yield stress typically decreases, as indicated in Fig. 1 [21,22].

Mode-coupling theorists suggested years ago that the colloidal glass transition and molecular glass transition are the same despite the fact that the control variables are different [23]. More recently, mode-coupling theories have been extended to include shear stress [24] or other control variables not derivable from Hamiltonians [25]. The jamming phase diagram suggests a reason why different jamming transitions might be related, independent of the validity of the modecoupling approximation.

While it has long been recognized that temperature, packing fraction, and stress can all control the stress relaxation time, the concept of the jamming phase diagram is a productive way to correlate jamming in different amorphous systems. The diagram implies that these three control parameters are important to all systems, so that one can study a single system as a function of all three variables. The diagram has proved to be a useful way to think about experiments, as shown recently by Trappe et al. [26] on solidification of attractive colloids. It also explicitly suggests new experiments to be done. For example, it suggests that one should measure how the relaxation time in a glass-forming liquid depends on applied stress. It also suggests that the introduction of a temperature to an otherwise jammed athermal system can help the system to flow. That is, temperature is a relevant variable for these transitions. This is, of course, in qualitative accord with the daily experience that shaking an otherwise jammed material can reinitiate flow. Perhaps the most significant implication of the diagram is that the jammed region might control the behavior nearby and that this is why different systems behave so similarly as they slow down on their approach to the jammed state.

\section{JAMMING AT POINT $\boldsymbol{J}$}

Perhaps the most daunting problem in studying any jamming transition is that the jammed surface depicted in Fig. 1 is typically not sharp, and is defined by the system's relaxation time exceeding experimental time scales. However, there is one point on the jamming phase diagram that is well defined [27], namely, the point labeled $J$ in Fig. 1. This point exists at zero temperature and zero applied shear stress for systems with repulsive, frictionless, finite-range potentials. This section is devoted to the special properties of point $J$.

\section{A. Method}

To explore point $J$, we have studied potentials of the following form:

$$
V\left(r_{i j}\right)=\left\{\begin{array}{lll}
\epsilon\left(1-r_{i j} / \sigma_{i j}\right)^{\alpha} / \alpha & \text { for } \quad r_{i j}<\sigma_{i j}, \\
0 & \text { for } \quad r_{i j} \geqslant \sigma_{i j},
\end{array}\right.
$$

where $\epsilon$ is the characteristic energy scale of the interaction, $r_{i j}$ is the separation between the centers of particles $i$ and $j$, and $\sigma_{i j}$ is the sum of the radii of particles $i$ and $j$. We study three different potentials, namely, $\alpha=2$ for repulsive harmonic springs, $\alpha=3 / 2$ for repulsive nonlinear springs that are harder than harmonic springs, and $\alpha=5 / 2$ for repulsive Hertzian interactions that are softer than harmonic springs [28]. It is important to note that the interactions are finite in range-particles do not interact unless they overlap. Potentials of this form were motivated by granular materials where particles have a well-defined diameter and do not interact except for a strong repulsive force that keeps the particles from deforming too much. In our two-dimensional (2D) simulations, we have used 50-50 mixtures of particles with a size ratio of 1.4 in order to prevent crystallization $[29,30]$. The diameter of the smaller particle is denoted by $\sigma$. In three dimensions (3D), we have studied the same bidisperse mixture as well as monodisperse systems with particle diameter $\sigma$. We have studied the finite-size effects by varying the number of particles in the sample between $4<N<4096$ in $2 \mathrm{D}$ and $3 \mathrm{D}$.

Of crucial importance is the protocol for the creation of configurations at $T=0$ and a given packing fraction $\phi$. To obtain such states, we start each simulation with a fixed number of particles $N$, with the particle positions chosen completely at random (this corresponds to $T=\infty$ ) within a square or cubic box with side length $L$ and periodic boundary conditions. Starting with randomly generated $T=\infty$ states guarantees that we sample all phase space equally. We then bring the system to the nearest potential-energy minimum by constantly moving downward on the potential energy surface. We do this using conjugate-gradient techniques [31]. Each conjugate gradient energy minimization is terminated when one of the following two stopping criteria is satisfied: (1) the total potential energy per particle satisfies $V / N<10^{-16}$ (this corresponds to a very small pressure, $p<10^{-10}$ ) and (2) $V / N$ for successive iterations deviates by less than $10^{-15}$. This procedure brings the system extremely close to $T=0$. Note that this procedure is identical to that for finding the "inherent structures" of the $T=\infty$ states [32].

In addition to studying the $T=0$ states generated by the protocol described above, we explore their properties by perturbing them slightly. We compress them, decompress them, or apply shear strains. After each infinitesimal perturbation, we can again employ the conjugate-gradient technique. Since this technique takes the system to the bottom of its local potential well, the quantities we measure in this way are related to the static, or infinite-time $(t=\infty)$, response (the static bulk or shear moduli $B_{\infty}$ or $G_{\infty}$ ) of the configurations. We have also measured the $t=0$ moduli $B_{0}$ and $G_{0}$ by measuring the response to a perturbation immediately after it has been applied (before minimizing the energy by the conjugate gradient technique). The shear and bulk moduli are obtained by measuring the response of the pressure tensor [33] 


$$
p_{\alpha \beta}=-L^{-d} \sum_{i>j} r_{i j \alpha} \frac{r_{i j \beta}}{r_{i j}} \frac{d V}{d r_{i j}}
$$

to shear and compression perturbations, where $r_{i j \alpha}$ is the $\alpha$ component of $\vec{r}_{i j}$ and $d$ is the dimensionality of the system. To measure the bulk modulus, we calculate $B=\phi d p / d \phi$, where the pressure is $p=\Sigma_{\alpha} p_{\alpha \alpha} / d$. To measure the shear modulus, we calculate $G=d \Sigma / d \gamma$, where $\Sigma=-p_{x y}$, after applying a shear strain in the $x$ direction with a strain gradient in the $y$ direction. The pressure $p$, stress $\Sigma$, bulk modulus $B$, and shear modulus $G$ are measured in units of $\epsilon / \sigma^{d}$, lengths are measured in units of $\sigma$, and time scales or inverse frequencies are measured in units of $\sigma \sqrt{m / \epsilon}$ where all particles have equal mass $m$.

\section{B. $J$ represents the onset of jamming for a single configuration}

It is important to note that each initial $T=\infty$ state can yield a different value of the packing fraction $\phi_{c}$, where the pressure and potential energy first become nonzero. Despite this ambiguity about the value of the threshold $\phi_{c}$, we find that there are robust results when we measure properties as a function of $\phi-\phi_{c}$, including scaling laws, that appear to be the same for all initial configurations. In Sec. II C, we will examine the nature of the distribution of these values of $\phi_{c}$. In this section, we will show that it is possible to locate a well-defined onset of jamming, $\phi_{c}$, for each initial state.

To test whether a given $T=0$ state is jammed or not, two separate criteria must be met: a jammed state must have a nonzero static (i.e., infinite time) value of both the bulk modulus and the shear modulus. As we show below, for each state that we have studied, the static bulk and static shear moduli approach zero at the same density $\phi_{c}$. Thus, $\phi_{c}$ specifies the onset of jamming for each state.

At $T=0$ and $\Sigma=0$, no two particles can interact if the density is low enough. If two particles were to overlap, their repulsive potentials would simply push them apart during the conjugate gradient energy minimization process until they no longer touched. Since there is neither thermal energy nor shear stress to compete with the particles' potential energy, they will never be forced back into contact. Thus, at sufficiently low densities, there are no particle overlaps and the final potential energy $V$ and the pressure $p$ are both zero so that the system has a zero static bulk modulus. At the threshold packing fraction $\phi_{c}$, particles just come into unavoidable contact since there is no longer enough free space to allow them to move apart. As the system is compressed further, the particles overlap, the energy and pressure are nonzero and the bulk modulus is nonzero because the pressure increases upon compression.

For each initial $T=\infty$ state, we first obtain a $T=0$ state using conjugate gradient minimization. For that $T=0$ state, we measure a precise value of $\phi_{c}$, as follows: If the configuration has zero pressure, we compress the system (by increasing the size of each particle by the same fixed fraction) in very small steps, applying conjugate-gradient energy minimization after each step, until the pressure becomes nonzero at $\phi_{c}$. Conversely, if the configuration has a nonzero

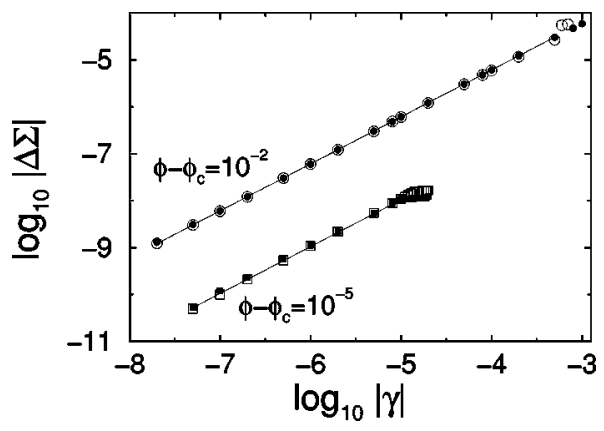

FIG. 2. The infinite-time stress $\Delta \Sigma=\Sigma(\gamma)-\Sigma(0)$ following an applied shear strain $\gamma$. The resulting stress-strain curve is linear for sufficiently small strains and independent of the sign of the strain. Open (filled) symbols indicate negative (positive) strains. These curves were generated using 3D monodisperse systems $(N=512)$ with harmonic repulsions. Circles and squares represent systems with packing fractions $\phi-\phi_{c}=10^{-2}$ and $10^{-4}$, respectively. The solid lines have slopes equal to 1 . The shear modulus, yield stress, and yield strain (where stress vs strain becomes nonlinear) tend to zero as $\phi$ approaches $\phi_{c}$, where $\phi_{c}$ is the onset of jamming for a given configuration.

pressure, we decompress the system in small steps, applying conjugate-gradient energy minimization after each step, until the pressure reaches zero at $\phi_{c}$. We insure that the system does not cross over any energy barriers during these procedures by compressing (or decompressing) in successively smaller increments. As the density variation is made finer and finer, we make sure that we end up in precisely the same configuration for all the particles independent of the size of the increment. Increments were in the range $\Delta \phi$ $=\left[10^{-6}-10^{-4}\right]$, with smaller increments used for smaller systems and systems closer to $\phi_{c}$.

At each packing fraction, we measure the static shear modulus $G_{\infty}$, by applying a very small shear strain, minimizing the energy with the conjugate gradient technique, and measuring the final induced stress. (Again, we insure that no energy barriers are crossed by applying successively smaller increments of shear strain. The strain increments were in the range $\left[5 \times 10^{-8}-10^{-5}\right]$ with smaller increments used for smaller systems and systems closer to $\phi_{c}$.) The shear modulus is calculated by measuring the linear relation between stress and strain, as shown in Fig. 2.

Figure 3 shows the results for the pressure $p$ as a function of $\phi-\phi_{c}$ for monodisperse systems in three dimensions using both harmonic $(\alpha=2)$ and Hertzian $(\alpha=5 / 2)$ potentials. We also include our earlier results for bidisperse systems in two and three dimensions using those same two potentials [27]. We find that the data for $p$ as a function of $\phi-\phi_{c}$ collapse onto a single curve for different initial states (each set of points corresponds to data from five different states). Thus, although each initial state has a different value of $\phi_{c}$, all states behave the same way as a function of $\phi-\phi_{c}$ when compressed above $\phi_{c}$.

In Fig. 4, we show the static shear modulus $G_{\infty}$ for the same initial states as shown for the pressure. Again, we find that data for different initial states collapse on a single curve when $G_{\infty}$ is plotted against $\phi-\phi_{c}$. Note that $\phi_{c}$ was deter- 


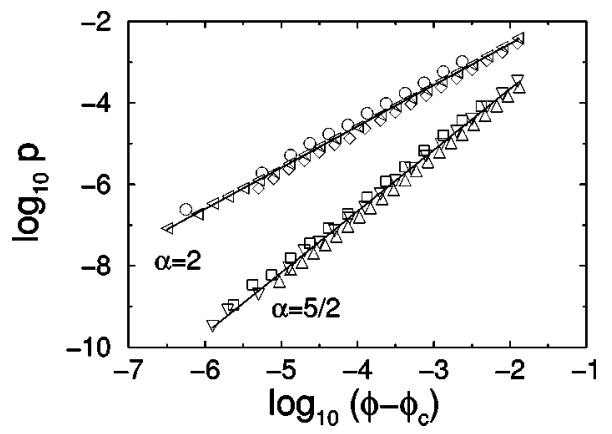

FIG. 3. Upper curves: Pressure $p$ vs $\phi-\phi_{c}$ for 3D monodisperse (circles), 3D bidisperse (diamonds), and 2D bidisperse (leftward triangles) systems with harmonic repulsions $(\alpha=2)$. The solid line has slope of 1.0. Lower curves: $p$ vs $\phi-\phi_{c}$ for 3D monodisperse (squares), 3D bidisperse (upward triangles), and 2D bidisperse (downward triangles) systems with Hertzian repulsions ( $\alpha$ $=5 / 2$ ). The solid line has a slope of 1.5 . These symbols for the different systems are used throughout the text. $N=1024(N=512)$ particles were used for the 2D (3D) systems.

mined by where the pressure approaches zero, not by where the static shear modulus first approaches zero. Thus, Figs. 3 and 4 show that the static shear modulus $G_{\infty}$ and the pressure $p$ (and therefore, the static bulk modulus $B_{\infty}$ as well) approach zero at the same packing fraction $\phi_{c}$ to a precision of better than two parts in $10^{5}$ for the monodisperse systems. Each state develops a bulk modulus and shear modulus at the same packing fraction. This is true for all polydispersities, dimensionalities, and potentials studied. Thus, $\phi_{c}$ truly marks the onset of jamming for a given initial state.

Note that in measuring the static shear modulus, we apply a shear stress in a given direction. Although we have shown that every studied state can withstand a shear stress in that direction for $\phi>\phi_{c}$, it is not obvious from these measurements that every state can withstand a shear stress in any arbitrary direction. To address this, we have studied the eigenvalues of the dynamical matrix [34] for our $T=0$ configurations with harmonic repulsions. We find that at least for

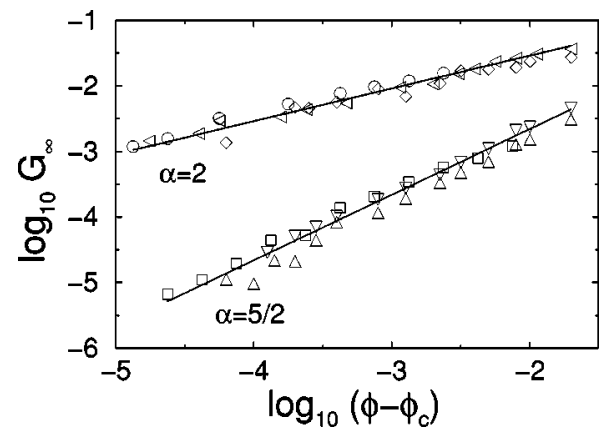

FIG. 4. Upper curves: Static shear modulus $G_{\infty}$ vs $\phi-\phi_{c}$ for 3D monodisperse (circles), 3D bidisperse (diamonds), and 2D bidisperse (leftward triangles) systems with harmonic repulsions ( $\alpha$ $=2$ ). The solid line has a slope of 0.5. Lower curves: $G_{\infty}$ vs $\phi$ $-\phi_{c}$ for 3D monodisperse (squares), 3D bidisperse (upward triangles), and 2D bidisperse (downward triangles) systems with Hertzian potentials $(\alpha=5 / 2)$. The solid line has a slope of 1.0. $N$ $=1024(N=512)$ particles were used for the $2 \mathrm{D}(3 \mathrm{D})$ systems.
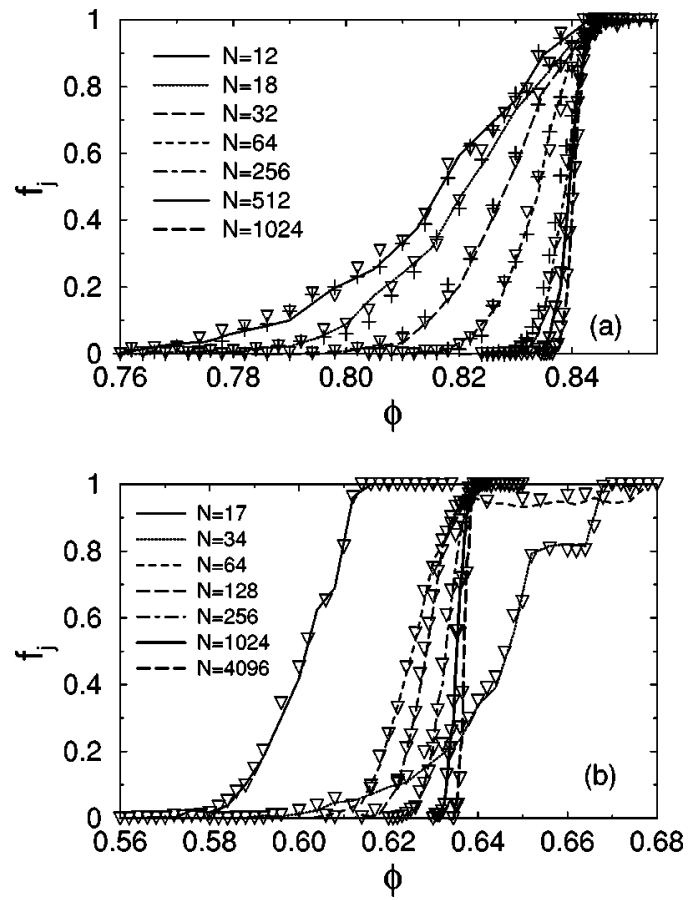

FIG. 5. Fraction $f_{j}$ of jammed states as a function of $\phi$ for (a) 2D bidisperse systems and for (b) 3D monodisperse systems with harmonic and Hertzian repulsions. In (a) and (b), the lines (downward triangles) represent potentials with $\alpha=2(\alpha=5 / 2) . f_{j}$ for 2D bidisperse systems with $\alpha=3 / 2$ are also shown in (a) using plus symbols. Each curve represents a different system size $N$.

$\phi-\phi_{c} \geqslant 10^{-6}$, the only zero-frequency modes correspond to isolated clusters of "rattlers," i.e., particles that do not overlap with any other particles and to uniform translations of the entire system. The lack of any nontrivial zero-frequency modes shows unambiguously that the system can withstand a shear stress in all directions. We will discuss the statistics of rattlers in greater detail in Sec. II E and the properties of the dynamical matrix in more detail in Sec. II G.

\section{Onset of jamming is sharp in the limit of infinite system size}

In the preceding subsection, we showed that different initial random $(T=\infty)$ states have inherent structures $(T=0$ states) that jam at different threshold values $\phi_{c}$. Here we measure the distribution of jamming thresholds. For each system size $N$ and packing fraction $\phi$, we start with at least 500 (100 for the largest system sizes) random $(T=\infty)$ configurations and use the conjugate gradient method to quench each configuration infinitely rapidly to $T=0$. We then find the fraction of these final states that are "jammed," i.e., that have a finite pressure and static shear modulus. The resulting fraction $f_{j}$ of jammed states is shown as a function of $\phi$ in Fig. 5(a) for a two-dimensional bidisperse system and in Fig. 5(b) for a three-dimensional monodisperse system with harmonic repulsions. Similar graphs were shown for threedimensional bidisperse systems with harmonic repulsions in Ref. [27].

In measuring these distributions, the system remains at one fixed, well-defined density since we do not dilate or 

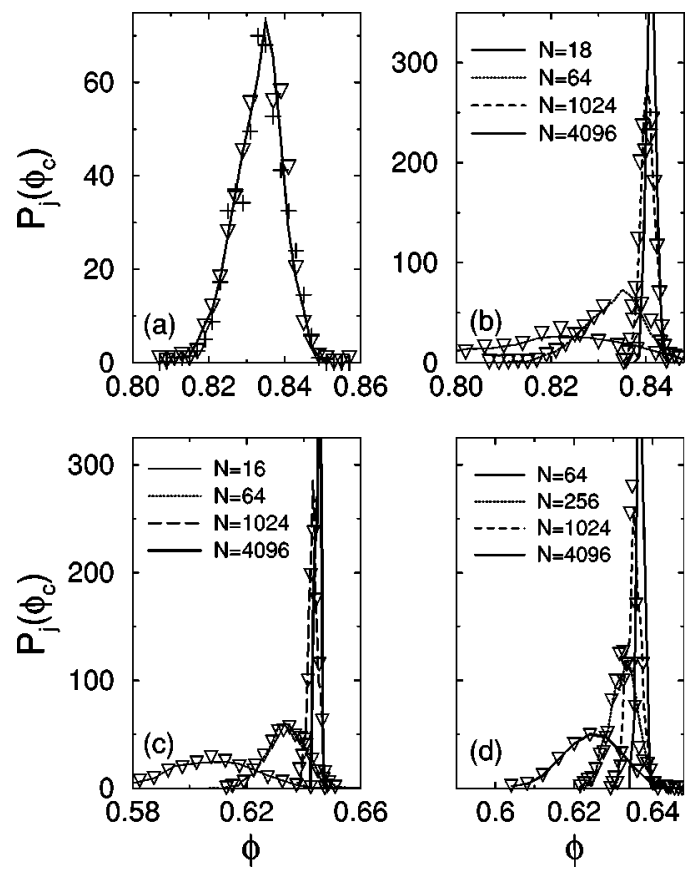

FIG. 6. (a) Distribution of jamming thresholds $P_{j}\left(\phi_{c}\right)$ for a 2D bidisperse system with $N=64$ for the three different potentials studied $\left(\alpha=3 / 2,2\right.$, and 5/2). $P_{j}\left(\phi_{c}\right)$ for (b) 2D bidisperse systems, (c) 3D bidisperse systems, and (d) 3D monodisperse systems with harmonic and Hertzian potentials for various system sizes. In (a)-(d), the pluses, lines, and downward triangles represent potentials with $\alpha=3 / 2,2$, and $\alpha=5 / 2$, respectively. The distributions for small 3D monodisperse systems $(N<64)$ were not shown in $(\mathrm{D})$ because we wanted to emphasize the monotonic behavior of the peak in $P_{j}\left(\phi_{c}\right)$ at large $N$.

shrink the particles. Also, during the quench itself, there are no dynamics. The system only travels on the potential energy surface and descends via the most rapid route to the nearest local potential-energy minimum. This distribution is therefore not a function of the dynamics used in obtaining the final configurations, but depends only on the fixed potentialenergy landscape. By starting with $T=\infty$ states, we are sampling configuration space uniformly. Thus, the result shown in Figs. 5(a) and 5(b) is a measure of the total fraction of configuration space (i.e., the probability) that belongs in the basins of attraction of final configurations that are jammed.

Figures 5(a) and 5(b) show that the fraction of jammed states depends sensitively on system size. For the 2D bidisperse system [Fig. 5(a)], the curves progressively sharpen with increasing $N$, eventually approaching a vertical jump. The 3D monodisperse system [Fig. 5(b)] shows a similar behavior for $N>64$. For smaller values of $N$, there is enough partial crystallization to produce additional structure in the curves.

We calculate the distribution of jamming thresholds $P_{j}\left(\phi_{c}\right)$ by differentiating the data in Fig. 5 with respect to $\phi$. We find that the distributions are insensitive to the interparticle potential used. This is illustrated in Fig. 6(a) for 2D bidisperse systems at a fixed system size $N=64$. In this figure, we overlay the distributions for $\alpha=5 / 2$ (Hertzian repulsions; downward triangles) and $\alpha=3 / 2$ (plus symbols) on top

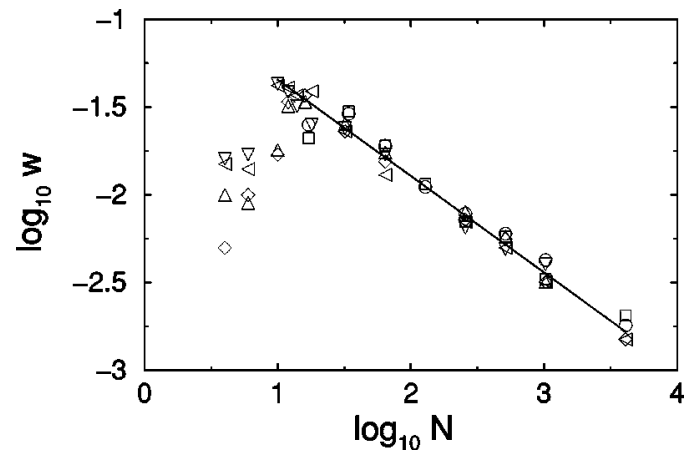

FIG. 7. Width of the distribution of jamming thresholds $w$ vs the number of particles $N$ for 2D bidisperse, 3D bidisperse, and 3D monodisperse systems with harmonic and Hertzian potentials. The solid curve has a slope of -0.55 . The symbols have the same meaning as in Fig. 3.

of the $\alpha=2$ (harmonic; solid lines) distributions. In Figs. 6(b) -6 (d), we overlay the distributions for $\alpha=5 / 2$ on top of those for $\alpha=2$ for all systems studied (2D bidisperse, 3D bidisperse, and 3D monodisperse) at several system sizes $N$. Within numerical error, the different potentials yield identical distributions at each $N$.

Figure 6 also shows that it is unlikely that a jamming threshold $\phi_{c}$ will be found at very low packing fraction, where almost all states are unjammed, or at very high packing fraction, where almost all states are already jammed. For small systems, the distributions are broad; as $N$ increases, they become sharper and taller. To quantify the change of the distributions with system size, we extract the full width at half maximum of the distribution, $w$, for each $N$. The results are plotted in Fig. 7 and are not monotonic in $N$. At very small $N$, there are only a few distinct configurations available to a static packing, so the distribution of jamming thresholds is narrow. The width grows with increasing $N$ to a maximum (near $N=10$ for bidisperse systems and near $N=30$ for 3D monodisperse systems). Above this value, the width decreases with increasing $N$. At the system size where the distributions are widest, $N \approx 10$ there is a reasonable probability of systems jamming at packing fractions as low as roughly $\phi=0.80$ in $2 \mathrm{D}$ bidisperse systems and $\phi=0.58$ in $3 \mathrm{D}$ bidisperse and monodisperse systems. Perhaps this is a coincidence, but it is interesting that the value in 3D corresponds to previous estimates of "random loose packing" from experiments [35]. It has been reported that hard particle methods (methods that strictly prohibit particle overlap) can produce jammed states with packing fractions that are much lower than the peak in the distribution of jamming onsets [36]. However, we have carried out similar hard particle simulations and find that these low- $\phi$ states are not jammed according to our definition given above. Instead, these states are unjammed and fall apart when they are slightly compressed or sheared.

In the large $N$ regime, Fig. 7 shows that the full width at half maximum of the distribution scales as

$$
w=w_{0} N^{-\Omega}
$$

with $\Omega=0.55 \pm 0.03$ and $w_{0}=0.16 \pm 0.04$ for all of the sys- 


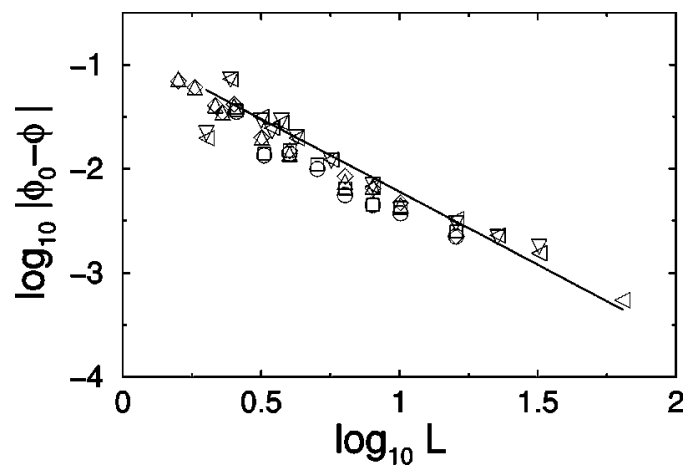

FIG. 8. Deviation of the peak in the distribution of jamming thresholds from its asymptotic value $\left|\phi_{0}-\phi^{*}\right|$ vs $L$ for 2D bidisperse, 3D bidisperse, and 3D monodisperse systems with harmonic and Hertzian potentials. The solid curve has a slope of $1 / \nu=1.40$. The symbols have the same meaning as in Fig. (3).

tems studied. This implies that as $N$ diverges, the width approaches zero and the distribution of jamming thresholds approaches a $\delta$ function. In other words, in the thermodynamic limit, essentially all of phase space jams at the same packing fraction $\phi^{*}$. This means that point $J$ in the jamming phase diagram is well defined as the onset of jamming.

\section{Point $J$ is random close packing in an infinite-size system}

Our results are relevant to hard-sphere systems because the $T=0$ configurations obtained by this protocol are allowed hard-sphere configurations if none of the particles overlap. Thus, at sufficiently low $\phi$, the conjugate gradient minimization technique will invariably yield allowed hardsphere states. Our protocol yields special insight into the nature of random close packing, a highly reproducible but heretofore somewhat vaguely defined state.

We make the connection to random close packing by asking what is the limiting $N \rightarrow \infty$ value of the jamming threshold $\phi^{*}$. We calculate it by extrapolating the peak positions $\phi_{0}$ of the distributions shown in Fig. 6 with respect to the system size. In Fig. 8, we plot the deviation of $\phi_{0}$ from $\phi^{*}$ as a function of $L \equiv N^{1 / d}$, where $d$ is the dimensionality. The peak position approaches its limiting asymptotic value as a power law in $L$ :

$$
\phi_{0}-\phi^{*}=\delta_{0} L^{-1 / \nu}
$$

By fitting the data to this form, we obtain $\nu=0.71 \pm 0.08$ and $\delta_{0}=0.12 \pm 0.03$ for all systems studied. Previously [27], we obtained $\phi^{*}$ for bidisperse systems in two and three dimensions. For monodisperse three-dimensional systems, we now find

$$
\phi^{*}=0.639 \pm 0.001
$$

We find that $\phi^{*}$ does not vary with potential; this follows from our result that the distributions of jamming thresholds are independent of potential $(\alpha=3 / 2, \alpha=2, \alpha=5 / 2)$ within the uncertainty of the measurement. Note that the value of $\phi^{*}$ in Eq. (5) for monodisperse three-dimensional systems is very close to what has been reported for random close pack- ing. Our value should be compared to other recent estimates of random close packing, $\phi_{r c p} \approx 0.64[37,38]$. This similarity is not a coincidence.

Random close packing cannot be defined in a mathematically precise way because the terms "random" and "close packed" are at odds with one another [38]. Because the close-packing density of an fcc packing is $\pi / \sqrt{18} \approx 0.74$ $>0.64$, one can always make the system more highly close packed (but less random) by introducing some degree of crystalline order. How random the system should be versus how close packed it should be is arbitrary. Torquato et al. therefore propose another term, "maximally random jammed state." By "jammed," they mean that any particle or set of particles cannot be translated relative to any of the rest of the particles in the system, and by "maximally random," they suggest a definition based on minimization of order parameters characterizing the extent of crystalline order, bond orientational order, etc. [39].

Here we suggest an alternate interpretation for random close packing using the language of a maximally random jammed state, but with different meanings attached to maximally random and jammed. In our case, the value $\phi^{*}$ is obtained by extrapolating the peak of the distribution of jamming thresholds to infinite system size. The peak of the distribution corresponds to the packing fraction with the maximum fraction of phase space (i.e., the maximum entropy) that belongs to the basin of attraction of jamming thresholds in the thermodynamic limit. We therefore propose that another way to define maximally random is by where the entropy of initial states is maximum, and that another way to define jammed is by the disappearance of zero-frequency modes of the dynamical matrix (with the exception of isolated clusters of rattlers). This definition has the advantage of avoiding the order parameter description, which will always be subject to uncertainty since one never knows if one has calculated the proper order parameter. It also provides a cleaner definition of the word jammed, since it depends on the nature of zerofrequency modes of the dynamical matrix. If one is testing whether a system is jammed by shifting particles, it is unlikely that one will hit on the exact combination of particle shifts that is characterized by the eigenvector of a zerofrequency mode. Finally, we note that our finding that virtually all initial states jam at the same value $\phi^{*}$ in the thermodynamic limit may explain why the value of random close packing is so robust despite the fact that it has not been well defined in the past. Although regions of the system can crystallize, such states are extremely rare and therefore unlikely to be observed for sufficiently large systems.

The above definition of random close packing, or the maximally random jammed state, is completely well defined for soft, finite-ranged repulsive potentials. What can be said about hard spheres? We can approach the hard-sphere limit by making the potential harder and harder, that is, by making the exponent in the potential, $\alpha$, [see Eq. (1)] approach 0 . Measuring $\phi^{*}$ as a function of $\alpha$ will then produce a limiting hard sphere, value for random close packing. Note that our results for $\phi^{*}$ are the same, within measurement error, for $\alpha=3 / 2, \alpha=2$ (harmonic), and $\alpha=5 / 2$ (Hertzian). Thus, the value of $\phi^{*}$ is insensitive to $\alpha$, suggesting that the hard- 
sphere limit of $\phi^{*}$ is the same as the value we have given in Eq. (5). Of course, it is not clear that the hard-sphere limit is well defined; different ways of taking the hard-sphere limit may lead to different results. If that is indeed the case, we would argue that hard spheres are a singular limit and thus unphysical. One should therefore concentrate on softer potentials for which unambiguous definitions can be constructed.

Another way that has often been employed to study hardsphere configurations near random close packing is to conduct density ramps. For example, in the LubachevskyStillinger algorithm [40], a hard-sphere system at low packing fraction is suddenly compressed (by increasing the radii of all the particles at some fixed rate) to a higher packing fraction. In the limit of infinite quench rate, one finds that the system jams at a random-close-packing density. One advantage to our protocol for systems with softer but still finite-ranged repulsive potentials is that, since the density is always held constant, we can quench the system to the final state within a fixed energy landscape. In the LubachevskyStillinger algorithm, the energy landscape changes throughout the density ramp because the density necessarily varies throughout the procedure.

One of the strengths of our procedure is that dynamics has no role. If we introduce dynamics by quenching the temperature of the system at some finite rate, we bias the distributions of jamming thresholds toward higher values of $\phi$. These distributions no longer represent features only of the potential-energy surface, but now also depend on dynamics through the quench rate. By contrast, our distributions are solely a geometric property of the potential-energy surface.

\section{E. Point $J$ is an isostatic point}

An isostatic configuration is defined by having the number of contacts in the system, $N Z / 2$, equal to the number of force balance equations [41], where $Z$ is the average number of contacts per particle. When this occurs, there is a unique solution for the forces between particles in a static packing, because the number of equations equals the number of unknowns. For purely repulsive, frictionless systems of spherical particles, the number of force balance equations is $N d$ so the isostatic condition is $Z=2 D$, where $d$ is the dimensionality of the system. We find [27] that there is a discontinuous

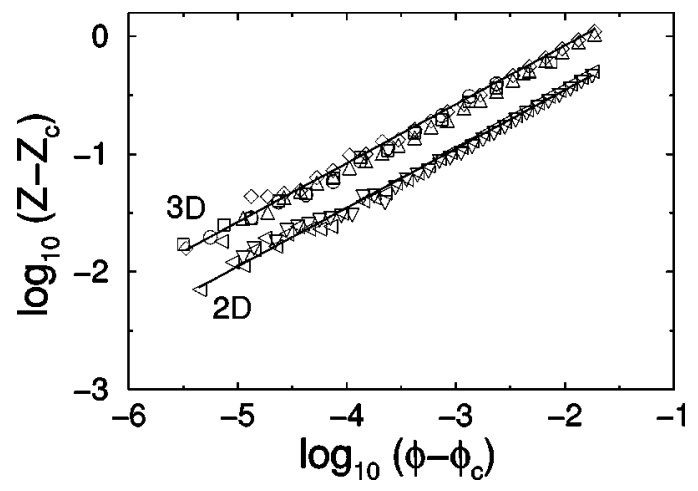

FIG. 9. Upper curves: Excess number of contacts per particle $Z-Z_{c}$ vs $\phi-\phi_{c}$ for 3D systems: monodisperse, harmonic (circles); monodisperse, Hertzian (squares); bidisperse, harmonic (diamonds); and bidisperse, Hertzian (upward triangles). Lower curves: $Z-Z_{c}$ vs $\phi-\phi_{c}$ for 2D systems: bidisperse, harmonic (leftward triangles) and bidisperse, Hertzian (downward triangles). $N=1024$ $(N=512)$ particles were used for the 2D (3D) systems. The symbols have the same meaning as in Fig. 3.

jump in $Z$ at the jamming threshold, $\phi_{c}$, of a given state. For $\phi=\phi_{c}^{-}$, there are no overlapping neighbors, $Z=0$, while for $\phi=\phi_{c}^{+}$there are $Z_{c}$ overlapping neighbors. The value of $Z_{c}$ can be obtained by measuring $Z$ at values just above $\phi_{c}$, as shown in Fig. 9. The straight lines in the plots are fits to the data of the form

$$
Z-Z_{c}=Z_{0}\left(\phi-\phi_{c}\right)^{\zeta}
$$

where $\zeta=0.50 \pm 0.03$ for all potentials, dimensions, and polydispersities studied.

As mentioned in Sec. II B, $\approx 5 \%$ of the particles are rattlers with no contacts at all, which do not contribute to the connected network. If we exclude the rattlers (so that we are only studying properties of the connected network) and assume $\zeta=0.5$, then we obtain precise values for $Z_{c}$, listed in Table I. These results are consistent with $Z_{c}=2 d$ in all cases, implying that the jamming threshold is an isostatic point. In the thermodynamic limit, $\phi_{c} \rightarrow \phi^{*}$, so point $J$ is an isostatic point. Note that our results for $Z$ show that point $J$ is the only point at which the packing is isostatic; above $\phi^{*}$, we find $Z>2 d$ so additional equations (the constitutive relations for

TABLE I. Coefficients and exponents for the power-law scaling of pressure $p$, shear modulus $G_{\infty}$, and coordination number $Z-Z_{c}$ for all systems studied.

\begin{tabular}{|c|c|c|c|c|c|c|c|c|c|}
\hline \multicolumn{10}{|c|}{ Power-law scaling } \\
\hline & \multirow{2}{*}{\multicolumn{2}{|c|}{ System }} & \multicolumn{7}{|c|}{ Quantity } \\
\hline & & & \multicolumn{2}{|c|}{$p$} & \multicolumn{2}{|c|}{$G_{\infty}$} & \multicolumn{3}{|c|}{$Z-Z_{c}$} \\
\hline $\mathrm{D}$ & Polydispersity & $\alpha$ & $p_{0}( \pm 0.05)$ & $\psi( \pm 0.03)$ & $G_{\infty}^{0}( \pm 0.05)$ & $\gamma( \pm 0.05)$ & $Z_{0}( \pm 0.5)$ & $\zeta( \pm 0.04)$ & $Z_{c}( \pm 0.03)$ \\
\hline 2 & $\mathrm{Bi}$ & 2 & 0.34 & 1.01 & 0.24 & 0.47 & 3.6 & 0.49 & 3.98 \\
\hline 2 & $\mathrm{Bi}$ & $5 / 2$ & 0.27 & 1.50 & 0.21 & 0.99 & 3.3 & 0.48 & 3.98 \\
\hline 3 & $\mathrm{Bi}$ & 2 & 0.28 & 1.03 & 0.21 & 0.48 & 8.4 & 0.47 & 5.98 \\
\hline 3 & $\mathrm{Bi}$ & $5 / 2$ & 0.18 & 1.51 & 0.17 & 1.02 & 7.4 & 0.49 & 5.98 \\
\hline 3 & Mono & 2 & 0.48 & 1.01 & 0.34 & 0.49 & 7.7 & 0.51 & 5.98 \\
\hline 3 & Mono & $5 / 2$ & 0.35 & 1.50 & 0.14 & 0.95 & 7.7 & 0.47 & 5.98 \\
\hline
\end{tabular}



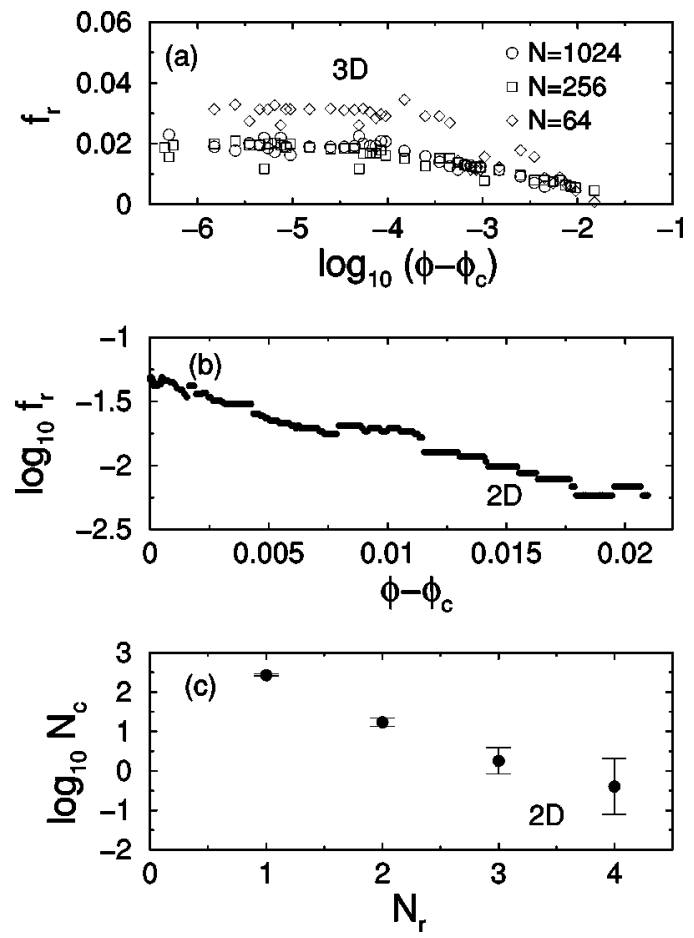

FIG. 10. (a) Fraction $f_{r}$ of particles that are rattlers as a function of $\phi-\phi_{c}$ for a 3D monodisperse system with harmonic repulsions at several system sizes $N$. (b) $f_{r}$ vs $\phi-\phi_{c}$ for a $N=1024$ bidisperse system with harmonic repulsions in 2D. (c) Number of clusters $N_{c}$ containing $N_{r}$ rattlers for five $N=100002 \mathrm{D}$ bidisperse systems with harmonic repulsions at $\phi-\phi_{c} \approx 10^{-2.5}$.

the particles, which depend on the potential used) are needed to solve for the forces between particles.

A more stringent condition for isostaticity is that the connected network (i.e., all particles in the system excluding rattlers) has no zero-frequency modes. As discussed in Sec. II B, we have looked for zero-frequency modes in packings above $\phi_{c}$, and have tested configurations with packing fractions as little as $10^{-6}$ above $\phi_{c}$. For all configurations tested, we have seen no zero-frequency modes except those associated with rattlers or with uniform translations. This suggests that point $J$ has no nontrivial zero-frequency modes.

We have studied the fraction $f_{r}$ of particles that are rattlers as a function of $\phi-\phi_{c}$ for both 2D bidisperse and 3D monodisperse systems with harmonic interactions. We show in Figs. 10(a) and 10(b) that the fraction of rattlers decreases with increasing packing fraction. We show in Fig. 10(a) that the fraction of rattlers is independent of system size for $N$ $>64$ in 3D. For the 2D bidisperse system, we have also studied the distribution of rattler cluster sizes. We find that most clusters have a single rattler and larger clusters are more rare. This is shown in Fig. 10(c).

\section{F. $g(r)$ diverges at point $J:$ A vanishing length scale}

A signature of jamming at point $J$ manifests itself in the pair correlation function $g(r)$. At this point, the particles just begin to touch so an important length scale-the distance between nearest-neighbor particles-goes to zero. This van-
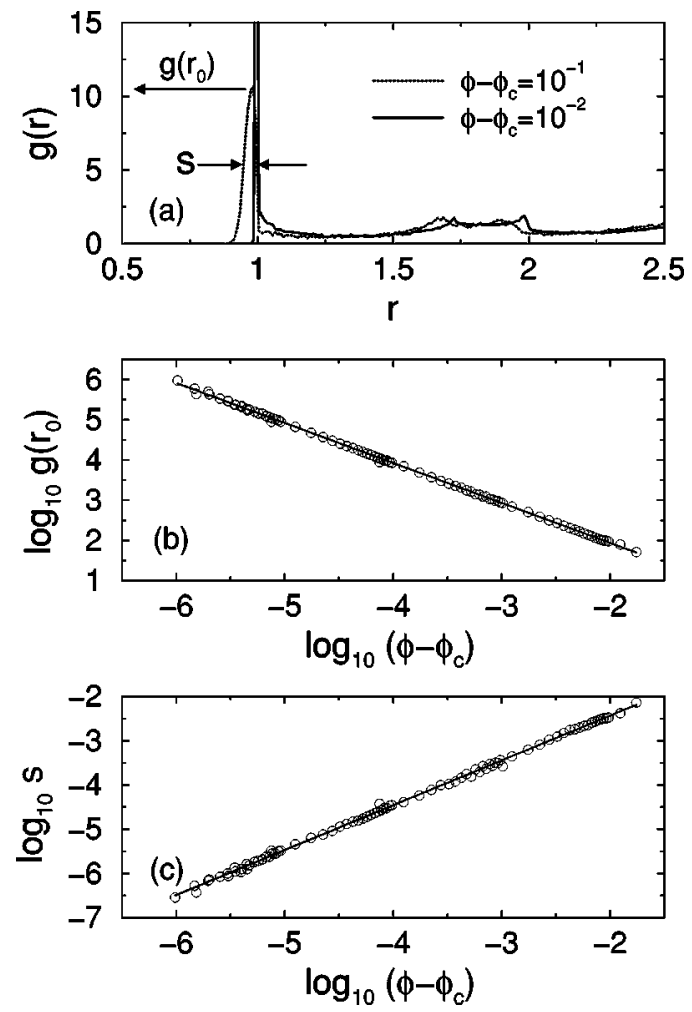

FIG. 11. (a) The radial distribution function $g(r)$ for a $N$ $=1024$ monodisperse system with harmonic repulsions in $3 \mathrm{D}$ at $\phi-\phi_{c}=10^{-1}$ and $10^{-2}$. The height of the first peak $g\left(r_{0}\right)$ and its left-hand width $s$ are defined. (b) Height of the first peak of $g(r)$ as a function of $\phi-\phi_{c}$ for the same system as in (a). The solid line has slope -1 . (c) Left-hand width $s$ of the first peak of $g(r)$ as a function of $\phi-\phi_{c}$ for the same system as in (a). The solid line has slope 1 .

ishing length scale gives rise to a divergence in $g(r)$ in the form of $\delta$ functions at $r=\sigma_{i j}$, the sum of the radii of neighboring particles. For simplicity, we will focus on monodisperse systems. Recall from Sec. II E that at $\phi_{c}^{-}$there are no contacts, whereas at $\phi_{c}^{+}, Z$ jumps to the isostatic value $Z_{c}$ $=2 d$. This discontinuity in $Z$ implies that there must be a $\delta$ function in $g(r)$ just at $\phi_{c}$ and that the area underneath this $\delta$ function must be exactly the coordination number at jamming: $Z_{c}=2 d$. This divergence is distinct from the divergence associated with the power-law increase above the first peak in $g(r)$ [where $g(r) \sim(1-r / \sigma)^{-1 / 2}$ as $r \rightarrow \sigma^{+}$[42]] since that power law is integrable whereas this one has a nonzero area.

Figure 11(a) shows $g(r)$ for a monodisperse, threedimensional system at two different values of $\phi-\phi_{c}$. Note that as $\phi$ approaches $\phi_{c}$ from above, the first peak grows higher and narrower. We can trace the evolution of the first peak by measuring its height as a function of $\phi-\phi_{c}$ [Fig. 11(b)]. We find that the height of the first peak at $r_{0}$ diverges as a power law:

$$
g\left(r_{0}\right)=g_{0}\left(\phi-\phi_{c}\right)^{-\eta}
$$

with $g_{0}=0.90 \pm 0.02$ and $\eta=0.993 \pm 0.002$. Previous hardsphere simulations [43] have measured, with much less pre- 
cision, the height of the first peak as $\phi_{c}$ is approached from below and found a similar exponent.

In Fig. 11(c), we plot the left-hand width at half-height of the first peak of $g(r)$ as a function of $\phi-\phi_{c}$. This width approaches zero as $\phi \rightarrow \phi_{c}^{+}$as a power law:

$$
s=s_{0}\left(\phi-\phi_{c}\right)^{\Delta},
$$

where $s_{0}=0.39 \pm 0.04$ and $\Delta=1.01 \pm 0.005$.

\section{G. There is an excess low-frequency contribution to the density of vibrational states at point $J$}

The normal modes of vibration provide a complete basis set to describe the motions of the particles in a jammed system. There have been many studies of normal modes in disordered systems [44-51]. In this section, we describe the normal mode spectrum as a function of packing fraction above $\phi_{c}$. A zero-frequency mode would indicate that some, possibly complicated, set of cooperative displacements of the particles could be made with no cost in energy. There should always be $d$ such modes corresponding to the simple uniform translation of the system for each of the $d$ dimensions. Every rattler particle will likewise contribute $d$ zero-frequency modes. If a configuration at $\phi=\phi_{c}$ is isostatic, as we claimed in Sec. II E, then above $\phi_{c}$ the only zero-frequency modes should be the trivial uniform translations of the entire system and the rattlers. As we mentioned above, we have found no other, nontrivial, zero-frequency modes. On the other hand, we must expect some change in the nature of the low-frequency modes as the packing fraction for a jammed configuration is lowered toward $\phi_{c}$. At this point, some extended mode or modes must approach zero frequency since it is precisely at $\phi_{c}$ that the system "falls apart" and becomes unjammed with $d N$ zero-frequency modes. How does the density of states evolve as $\phi-\phi_{c}$ approaches zero? In order to compute the normal modes and frequencies $\omega$ of the system, we diagonalize the dynamical matrix of the system [31]. The eigenvalues are the squares of the frequencies and the eigenvectors are the polarization vectors of the particles in each mode.

As in a crystal one expects the low-frequency excitations to be the long-wavelength sound (longitudinal and transverse) modes. This assumption gives a density of normal mode frequencies, $D(\omega)$, proportional to $\omega^{d-1}$. An earlier simulation [46] found an increase in the low-frequency density of states as the number of nearest neighbors in a glass was reduced. As we will show, our present results support this claim. In the previous study [46], nearest-neighbor bonds were severed at random with some probability. Here, we control the number of overlaps by varying the packing fraction, and we can reduce $Z$ all the way down to the isostatic value by approaching $\phi_{c}^{+}$. In Fig. 12, we show the density of states obtained for a monodisperse harmonic system in three-dimensions with $N=1024$ particles at $T=0$. Figure 12(a) contains the familiar result for compressed systems with packing fractions $\phi$ that are far above $\phi_{c}$. The largest $\phi-\phi_{c}$ studied is comparable to typical liquid densities in an equivalent Lennard-Jones system [52]. For the
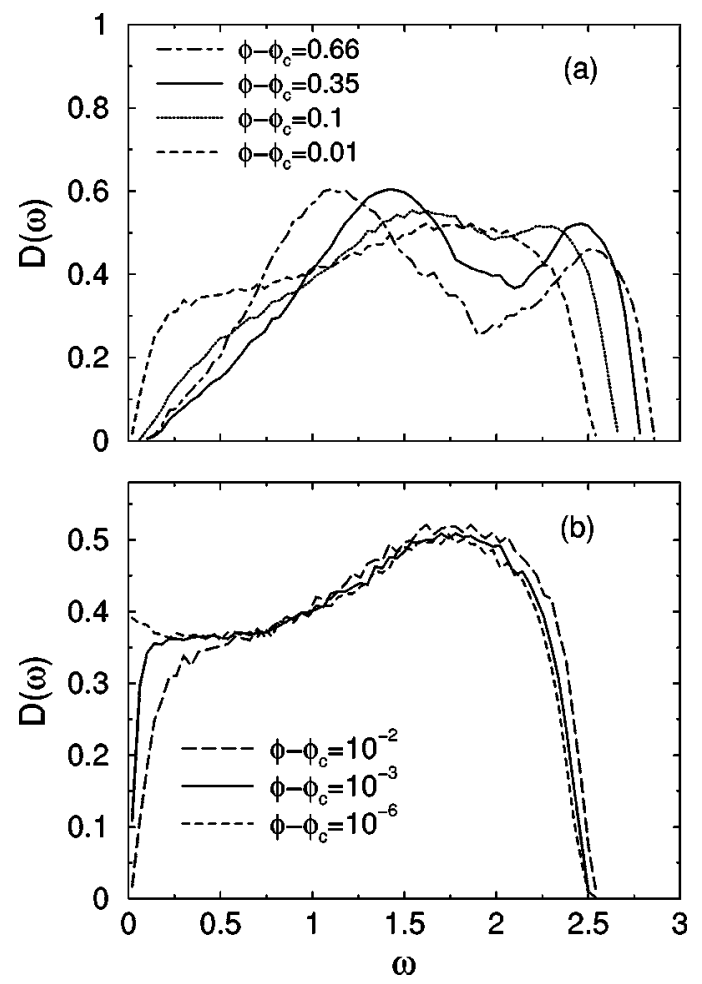

FIG. 12. Density of states $D(\omega)$ vs $\omega$ for a $3 D N=1024$ system with harmonic repulsions at packing fractions (a) far from $\phi_{c}$ and (b) close to $\phi_{c}$.

highest packing fractions, we see that there is an identifiable region where $D(\omega)$ increases as $\omega^{2}$, as expected. As the packing fraction is lowered, however, we see that the region of $\omega^{2}$ behavior shrinks, reminiscent of the results found in Ref. [46]. In Fig. 12(b), we show the behavior of $D(\omega)$ as $\phi$ approaches $\phi_{c}$ more closely. For this 1024-particle system, we see no sign of an $\omega^{2}$ region when $\left(\phi-\phi_{c}\right) \leqslant 0.1$. This region has presumably been pushed to low frequencies that are inaccessible in a system of this size because the excitations would have wavelengths that exceed the linear size of the system. Even though there is no $\omega^{2}$ behavior at $\phi-\phi_{c}$ $=0.1$, Fig. 12(b) shows that $D(\omega)$ drops as $\omega$ goes to zero. However, as $\phi-\phi_{c}$ decreases still further, this drop in $D(\omega)$ disappears. By $\phi-\phi_{c}=10^{-6}$, there is no evidence of it at all and $D(\omega)$ appears to approach a constant at zero frequency. This striking result is unanticipated. As the packing fraction is lowered, the density of states approaches a limiting, constant, nonzero value, instead of vanishing as expected for long-wavelength sound modes. Thus, there is a proliferation of anomalous low-frequency modes as point $J$ is approached from above.

\section{H. Power-law scaling near point $\boldsymbol{J}$}

So far, we have discussed a number of quantities that scale as power laws with $\phi-\phi_{c}$ as the jamming threshold is approached from the high-density side. Such quantities include pressure $p$ (Fig. 3), static shear modulus $G_{\infty}$ (Fig. 4), and coordination number, $Z-Z_{c}$ (Fig. 9). In addition, we have shown that the width $w$ (Fig. 7), and peak position $\phi_{0}$ (Fig. 8), of the distribution of jamming thresholds display 
power-law scaling with system size. Here we discuss the power-law exponents and their implications.

Figure 3 shows that the pressure vanishes as a power law as $\phi \rightarrow \phi_{c}^{+}$:

$$
p=p_{0}\left(\phi-\phi_{c}\right)^{\psi}
$$

The values for $p_{0}$ and $\psi$ are listed in Table I. Our results for $\psi$ are consistent with

$$
\psi=\alpha-1,
$$

independent of polydispersity or dimensionality.

The static shear modulus scales as

$$
G_{\infty}=G_{\infty}^{0}\left(\phi-\phi_{c}\right)^{\gamma},
$$

where $G_{\infty}^{0}$ and $\gamma$ are listed in Table I. The results are consistent with

$$
\gamma=\alpha-3 / 2,
$$

independent of polydispersity or dimensionality.

As discussed earlier in Sec. II E, the coordination number $Z-Z_{c}$ scales as a power law with $\phi-\phi_{c}$ [see Eq. (6)] with an exponent consistent with

$$
\zeta=1 / 2,
$$

independent of potential, dimensionality, and polydispersity. This result is consistent with earlier estimates from simulations in both 2D and 3D [27,42,53,54].

The height of the first peak of $g(r)$ scales as a power law:

$$
g\left(r_{0}\right)=g_{0}\left(\phi-\phi_{c}\right)^{-\eta}
$$

with $\eta=0.993 \pm 0.002$. This result was obtained for a threedimensional monodisperse system with harmonic repulsions. Similarly, the left-hand width of the first peak of $g(r)$ scales as a power law:

$$
s=s_{0}\left(\phi-\phi_{c}\right)^{\Delta},
$$

where $\Delta=1.01 \pm 0.005$.

Finally, recall the form of the fits to the width and peak position of the jamming threshold distributions, Eqs. (3) and (4), where the width scales as $w \sim N^{-\Omega}$ and $\phi^{*}-\phi_{0}$ $\sim L^{-1 / \nu}$. Figure 7 shows that $\Omega$ appears to be independent of potential, polydispersity, and dimensionality. We find $\Omega$ $=0.55 \pm 0.03$, consistent with

$$
\omega=1 / 2
$$

For the peak position, Fig. 4 shows that $\nu$ is independent of potential, polydispersity, and dimensionality. We find $\nu$ $=0.71 \pm 0.08$, consistent with

$$
\nu=2 / 3 .
$$

\section{Interpretation of power-law exponents}

Some of the exponents for the scalings with $\phi-\phi_{c}$ are straightforward to understand, while others are, as yet, without explanation.

Pressure and bulk modulus. The exponent for pressure, $\psi \approx \alpha-1$, can be explained if the system responds perfectly affinely to compression. If the deformation is affine, one would expect the exponent for the pressure to be the same as for the force law; this argument yields $\psi=\alpha-1$. Similarly, we would expect the bulk modulus to behave as a power law:

$$
B \sim\left(\phi-\phi_{c}\right)^{\beta},
$$

with $\beta=\alpha-2$, because the bulk modulus is related to the derivative of pressure with respect to packing fraction. We can check to see if the response of the packing to compression is truly affine by comparing the zero-time bulk modulus $B_{0}$ to the infinite-time, or static, bulk modulus $B_{\infty}$. To obtain $B_{0}$, we apply a compression (or expansion) and measure the change of pressure without allowing any of the particles to relax their positions. By construction, the compression (expansion) is perfectly affine throughout the sample because we increase (decrease) the radii of all of the particles by the same fixed fraction. (This is different from how one compresses a sample in a laboratory experiment, where the perturbation is applied at the boundaries of the sample.) To obtain $B_{\infty}$, on the other hand, we first apply the affine compression (or expansion), then allow the particles to shift their positions by minimizing the energy using the conjugate gradient technique. If the response to compression is perfectly affine, then the particles will not shift during the conjugate gradient process because the energy is already a minimum. In that case, we would expect $B_{\infty}=B_{0}$. The results are shown in Fig. 13. For all potentials, polydispersities, and dimensions studied, we consistently find that $B_{\infty}<B_{0}$, but that they both scale with the same power, consistent with $\beta$ $=\alpha-2$. These results show that nonaffine deformations due to disorder in the packing do reduce the coefficient of the scaling of the bulk modulus, but do not change the exponent. It is not obvious why the exponent is unchanged.

Shear modulus. Like the bulk modulus, the shear modulus is also given by two derivatives of the energy. However, we do not find that the scaling exponent for the static shear modulus $\gamma$ satisfies $\gamma=\alpha-2$. Rather, we find $\gamma \approx \alpha-1.5$ [see Eq. (12)]. To gain insight into this discrepancy, we have examined the zero-time shear modulus $G_{0}$ as well as the static or infinite-time shear modulus $G_{\infty}$. As with the bulk modulus, to measure $G_{0}$ we first apply an affine shear strain and measure the resulting stress without allowing any of the particles to shift their positions. To measure $G_{\infty}$, on the other hand, we apply the conjugate gradient technique once the affine shear is applied and measure the resulting stress after the energy has been minimized. Since the shear modulus is the second derivative of the energy, we would expect the exponent for $G_{0}$ to be $\gamma_{0}=\alpha-2$. This is indeed what we find, as shown in Fig. 14. The figure shows that $G_{\infty}<G_{0}$, as expected; the system relaxes to a lower value of the shear stress than it has initially. Although Lacasse et al. [21] have previously pointed out that nonaffine deformations can re- 

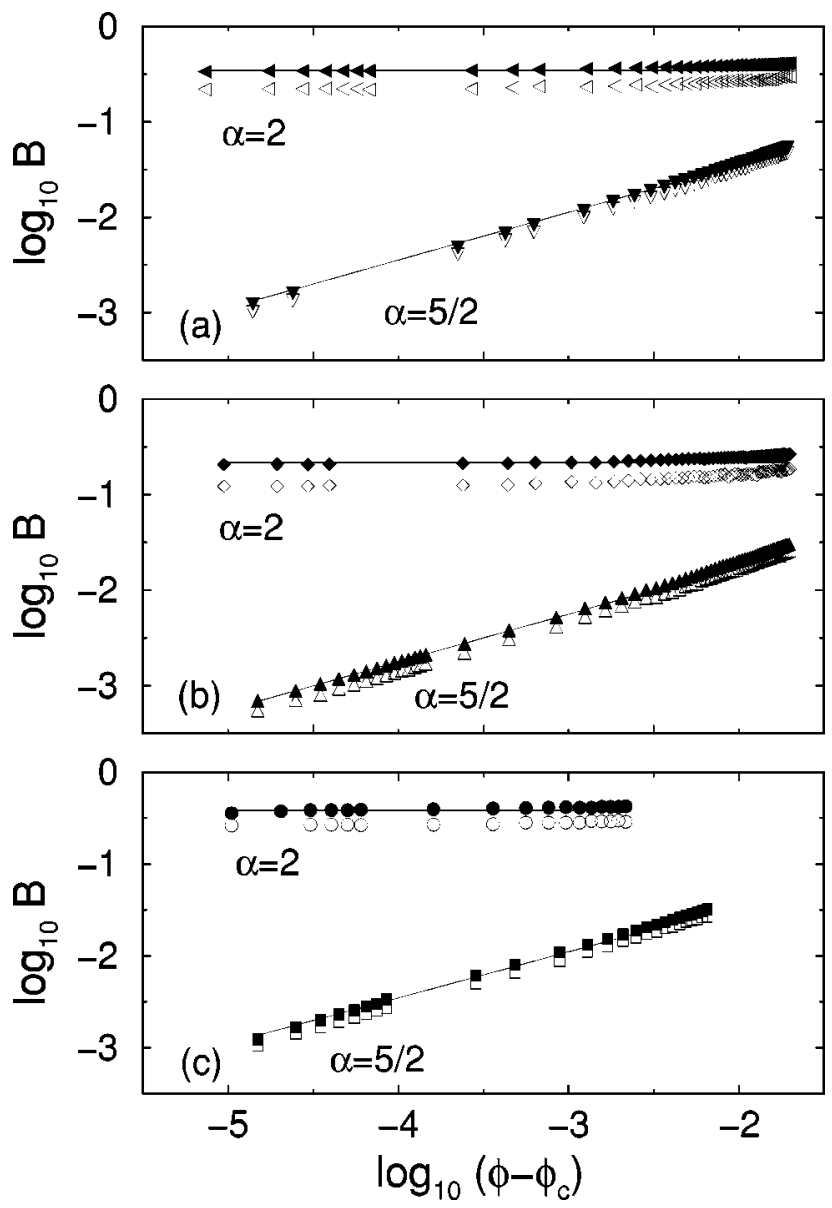

FIG. 13. Zero-time $\left(B_{0}\right)$ (closed symbols) and infinite-time ( $B_{\infty}$ ) (open symbols) bulk moduli vs $\phi-\phi_{c}$ for (a) 2D bidisperse systems, (b) 3D bidisperse systems, and (c) 3D monodisperse systems with harmonic and Hertzian potentials. The solid curves have slopes equal to 0 and 0.5. $N=1024(N=512)$ particles were used for the $2 \mathrm{D}(3 \mathrm{D})$ systems.

duce the shear modulus in emulsions, they did not show that the effect of these deformations would be to produce a power-law dependence of the shear modulus upon compression. Our results show that such a power-law scaling exists for the static shear modulus and that the effect of the nonaffine deformations is to shift the value of the exponent from $\gamma=\alpha-2$ (appropriate to the $t=0$, affine situation) to $\gamma \approx \alpha$ $-3 / 2$ (appropriate to the $t=\infty$ case where all relaxation has been allowed to take place). The effect of nonaffine deformation is much more pronounced for the shear modulus than it is for the bulk modulus. In the latter case, the power-law exponent remained unaffected and only the prefactor was changed. In the case of the shear modulus, the nonaffine deformation changes the scaling exponent as well as the prefactor. As the critical density $\phi_{c}$ is approached from above, the nonaffine deformations play a larger and larger role so that $G_{0} / G_{\infty}$ diverges at $\phi_{c}$.

Coordination number. Figure 9 shows that the coordination number scales as $Z-Z_{c} \sim\left(\phi-\phi_{c}\right)^{\zeta}$, where $\zeta$ is independent of potential, polydispersity, and dimensionality. The fact that $\zeta$ is independent of potential is intriguing because it suggests that $\zeta$ depends only on the geometry of the packing.

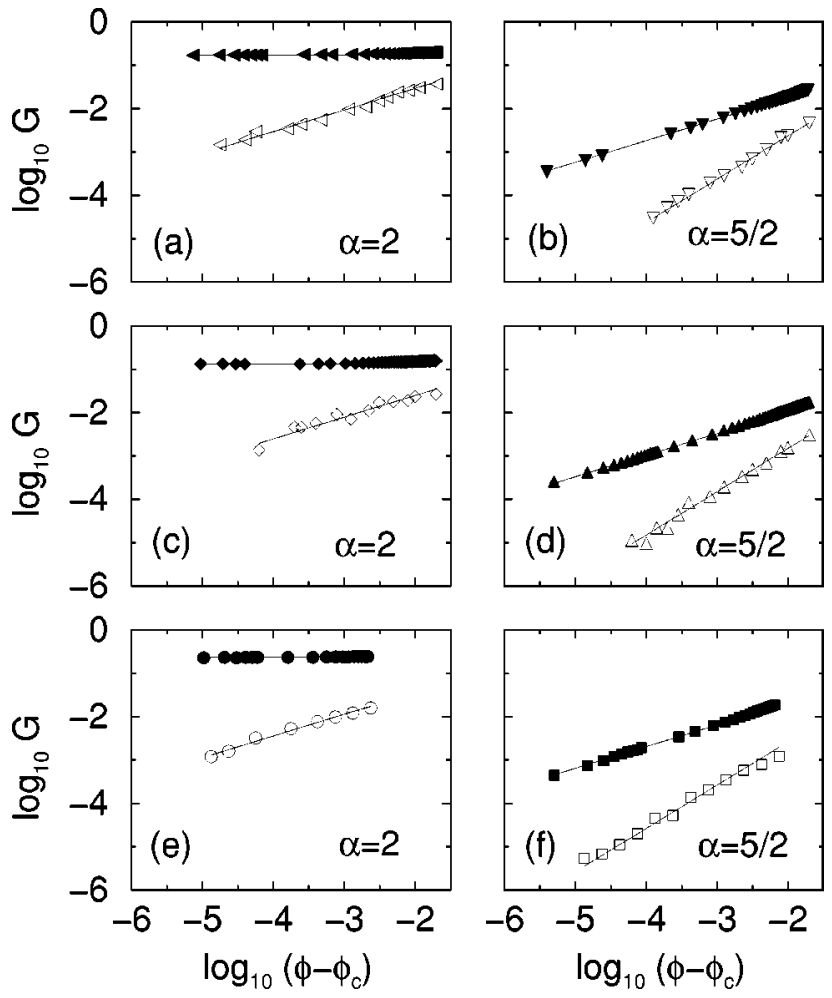

FIG. 14. Zero-time $\left(G_{0}\right)$ (filled symbols) and infinite-time $\left(G_{\infty}\right)$ (open symbols) shear moduli vs $\phi-\phi_{c}$ for (a) and (b) 2D bidisperse systems, (c) and (d) 3D bidisperse systems, and (e) and (f) 3D monodisperse systems. Harmonic and Hertzian repulsions are labeled $\alpha=2$ and $\alpha=5 / 2$, respectively. In (a), (c), and (e), the solid curves have slopes equal to 0 and 0.5 . In (b), (d), and (f), the solid curves have slopes equal to 0.5 and 1.0. $N=1024(N=512)$ were used for the 2D (3D) systems.

The fact that $\zeta$ is also independent of dimensionality suggests that there is a property of the packing that is independent of $d$.

Recent results [42] for the pair correlation function $g(r)$ of three-dimensional harmonic packings slightly below the jamming threshold show that $g(r)$ contains a power-law region near $r=\sigma$, where $\sigma$ is the sphere diameter:

$$
g(r) \propto(1-r / \sigma)^{-1 / 2}
$$

If one assumes an affine deformation upon compression, consistent with the scaling results for pressure and bulk modulus, then one of the consequences of Eq. (19) is that the coordination number should increase with the power $\zeta$ $=1 / 2$, as we have observed. Thus, the scaling in Eq. (19) is consistent with our result $\zeta=1 / 2$. The origin of both results, however, is still not understood.

Height and width of first peak of $g(r)$. We find that the height of the first peak of $g(r)$ diverges with an exponent $\eta \approx 1$ [see Eq. (14)] and that the left-hand width of the first peak vanishes with an exponent $\Delta \approx 1$ [see Eq. (15)] as $\phi$ $\rightarrow \phi_{c}^{+}$. The fact that $\eta \approx \Delta$ is consistent with our expectation that the area of the first peak is roughly $Z_{c}$. 


\section{Discussion of finite-size scaling exponents}

We have found that there are very strong system-size effects. As $N$ diverges, the width of the distribution of jamming thresholds vanishes as $N^{-\Omega}$, leaving a $\delta$-function distribution at point $J$. We find that $\Omega$ is very close to $1 / 2$ [see Eq. (16)]. It is not obvious that this result can be explained by a simple central limit theorem argument because the packing density is a subtle property of the packing geometry. Independent of the explanation for this exponent, there are still correlations extending across the entire system once it is jammed.

The peak position shifts toward the random-close-packing density as $L^{-1 / \nu}$. This result suggests that there is a long length scale appearing in the problem near the onset of jamming, which scales as $\left(\phi-\phi_{c}\right)^{-\nu}$. Note that our result $\nu$ $=0.71 \pm 0.08$ is a typical value for a correlation length exponent.

\section{Lack of self-averaging at point $\boldsymbol{J}$}

At point $J$, there is no self-averaging in the sense that the average properties of a very large system are not the same as the average over an ensemble of many smaller systems at the same packing fraction. This property can be understood by considering a system of size $N$ and the behavior as $N$ diverges. For a finite-sized system, Fig. 6 shows that there is a distribution of jamming thresholds $\phi_{c}$. Consider a given packing fraction $\phi$ which is within this distribution. Some of the configurations at this $\phi$ will be jammed, and others will be unjammed with $p=0$. For an unjammed configuration $p$ $=0$ for every subregion of the configuration, as well. (This is exact even in the infinite system-size limit.) However, at the same $\phi$, there will exist jammed configurations for which $p>0$. For those configurations, we have found $p>0$ for almost all subregions. There are only small clusters of rattlers that have zero local pressures. The number of such clusters decreases rapidly with the size of the cluster [see Fig. 10(c)]. Thus, the value of the pressure averaged over all configurations cannot be the same as the value of the pressure averaged over an arbitrary given configuration. As a result, there is no self-averaging. As the system size $N$ increases, the distribution of jamming thresholds narrows. As a result, the lack of self-averaging will be observed over a smaller region of $\phi$ that eventually narrows to a point (point $J$ ) in the infinite $N$ limit.

The lack of self-averaging is evident in the distribution of interparticle normal forces between particles, $P(F)$ [27]. For a given configuration, the average interparticle force $\langle F\rangle$ is directly proportional to the pressure of that configuration as shown in Fig. 15 for a 3D monodisperse system with harmonic repulsions. Depending on whether one normalizes the forces in a given configuration to $\langle F\rangle$, the average within that configuration, and then averages $P(F /\langle F\rangle)$ over many configurations, or whether one normalizes the forces of all configurations to the same global average force $\langle\langle F\rangle\rangle$, and then calculates $P(F /\langle\langle F\rangle)$, one will get a different distribution function. This is shown in Fig. 16 for a 3D monodisperse system with harmonic repulsions. Note that the difference between $P(F /\langle F\rangle)$ and $P(F /\langle F\rangle\rangle)$ is largest near $\phi$

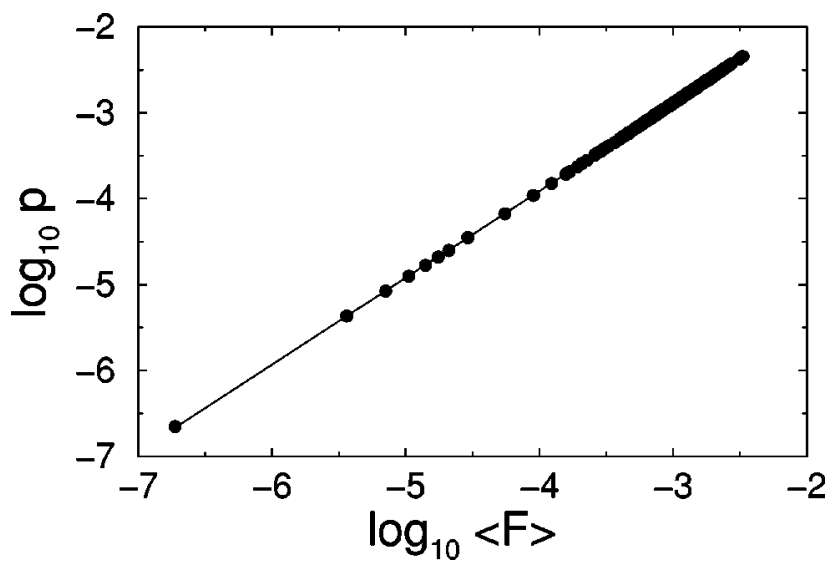

FIG. 15. Pressure $p$ vs average interparticle force $\langle F\rangle$ for a 3D monodisperse system $(N=512)$ with harmonic repulsions. The solid line has slope equal to 1 .

$=0.636$, which is near the peak $\phi_{0}$ of the distribution of jamming thresholds for the three-dimensional system shown $(N=1024)$. As the packing fraction is increased above $\phi_{0}$, the curves for $P(F /\langle F\rangle)$ and $P(F /\langle\langle F\rangle\rangle)$ look more and more similar. This is consistent with the argument above that the lack of self-averaging is most pronounced near the peak of the distribution of jamming thresholds. A simple argument for the shape of the tail of $P(F /\langle\langle F\rangle\rangle)$ was given earlier [27].
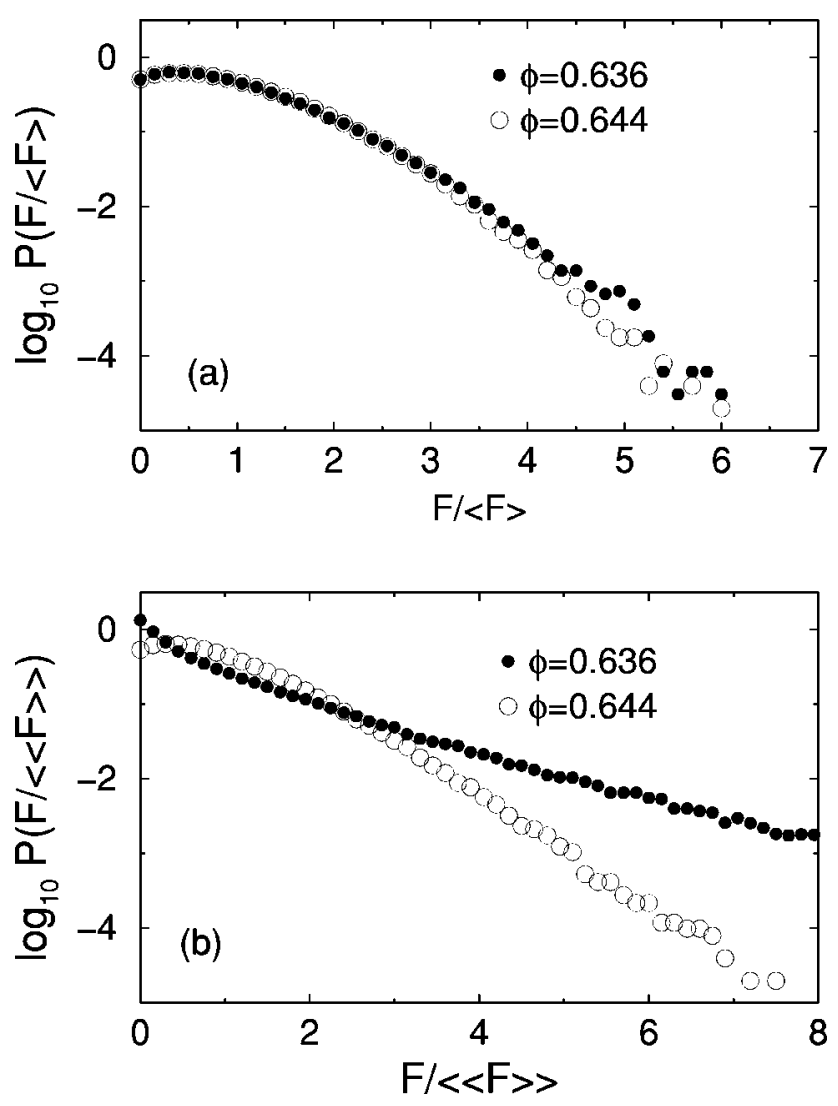

FIG. 16. Distribution of interparticle normal forces for a 3D monodisperse $(N=1024)$ system with harmonic repulsions. (a) $P(F /\langle F\rangle)$ vs $F /\langle F\rangle$ and (b) $P(F /\langle\langle F\rangle\rangle)$ vs $F /\langle\langle F\rangle\rangle$. 


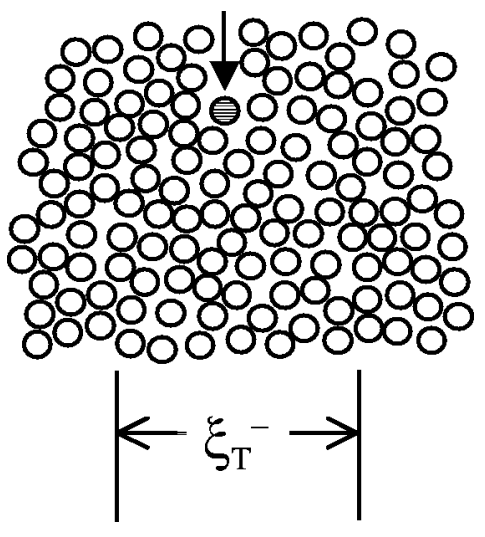

FIG. 17. Sketch of the transverse length scale.

\section{J. Critical behavior near point $J$}

In many ways, point $J$ resembles a critical point. We have shown in Figs. 3, 4, 9, 11, 13, and 14 that there is power-law scaling near point $J$ of quantities such as the pressure, shear modulus, bulk modulus, coordination number, and the height and width of the first peak of the pair correlation function [27]. We have also shown in Figs. 7 and 8 that there is finite-size scaling since the width and peak position of the distributions of jamming thresholds scale with the size of the system. This is reminiscent of behavior near an ordinary critical point. Finally, we demonstrated in Fig. 16 that properties, such as the force distribution, do not self-average near point $J$. As the system size increases, the packing fraction must be tuned closer and closer to the peak of the distribution of jamming thresholds in order to see the breakdown of self-averaging. This is also what one expects near an ordinary critical point, where the temperature must be tuned closer and closer to the critical point as the system size increases in order for the correlation length to exceed the system size.

The lack of self-averaging near point $J$ and the power-law scaling of the width and peak position of the jamming threshold distribution with system size, all suggest that there is a correlation length that diverges at point $J$. What might this length scale be? We speculate that there is a transverse length scale that does diverge as point $J$ is approached from below. If the system is held at a packing fraction slightly below the critical value, the system is unjammed and the particles can all move and rearrange. However, the number of particles that must move in order to allow a rearrangement will depend on how close one is to the transition. Thus, in an infinite system, if one applies a fixed, infinitesimal velocity to a single particle, we would expect the particle to disturb the surrounding particles as it moves. This disturbance will extend to a distance $\xi_{T}^{-}$, the transverse length scale, in a direction perpendicular to the applied force. We expect that $\xi_{T}^{-}$will diverge as one gets close to the transition because as the density approaches the close-packing value, more and more particles must rearrange to allow for the single-particle motion in the longitudinal direction. The idea behind this transverse length scale is shown in Fig. 17. Similar ideas are currently being explored experimentally in granular systems with friction [55] and in colloidal systems [56,57].
One might estimate the transverse length scale by computing how many particles must move laterally in order to insert an extra particle. This is the parking lot model [58]. According to this argument, the transverse length scale should diverge as $\xi_{T}^{-} \sim\left(\phi_{c}-\phi\right)^{-1 /(d-1)}$, for $\phi<\phi_{c}$, where $d$ is the dimensionality. We note, however, that this result does not agree with the correlation length exponent that we obtained from the finite-size scaling analysis [see Eq. (17)], which appears to be independent of dimensionality.

Although point $J$ resembles a critical point, it has properties unlike any other critical point ever studied. The exponents appearing in the scaling relations are independent of dimension, but do depend on the potential. The former observation could be reconciled with a normal critical point if the upper critical dimension for jamming was less than 2, but then we would not expect different potentials to yield different exponents. Likewise, if each different potential was in a different universality class and yielded different exponents, then the upper critical dimension should be above 3. There are other properties of point $J$ which are unusual (although not unheard of) for a critical point. At packing fractions below point $J$, the pressure, shear modulus, and contact number are all zero and the energy is zero everywhere. There are no fluctuations in these quantities, even infinitesimally close to point $J$, as $\phi \rightarrow \phi^{*}$ from below. In addition, there is a discontinuous jump in the value of the coordination number $Z$ from zero to $Z_{c}$ at $\phi^{*}$. We also note that we have identified a length scale that goes to zero at this point: the spacing between particles that form the connected network in the jammed state. This is seen in the divergence of the first peak of $g(r)$ (Fig. 11). At a critical point, one expects a single divergent length scale and not a length scale going to zero.

Perhaps, the most disturbing feature of point $J$, from the point of view of ordinary critical phenomena, is the difference in the behavior at fixed pressure and fixed volume. At fixed volume, we observe a finite-size rounding of powerlaw scaling and finite-size effects such as the lack of selfaveraging. This is because different states have different jamming thresholds, $\phi_{c}$. At a fixed $\phi$, different states are averaged together and the clean power-law behavior we observe as a function of $\phi-\phi_{c}$ will be rounded. However, Fig. 3 shows that a fixed pressure corresponds to a fixed value of $\phi-\phi_{c}$. When we plot quantities as a function of $\phi-\phi_{c}$, we do not see finite-size rounding of power-law behavior. Even for a finite-sized system, the behavior of the shear modulus, etc. appears to be a clean power-law down to the smallest measurable values of $\phi-\phi_{c}$. Thus, we do not see measurable finite-size effects at fixed pressure. The divergence of $g(r)$ also occurs even for a finite-sized system. These results are very different from what one would expect for an ordinary critical point.

It has been suggested also that point $J$ might correspond to the onset of rigidity percolation [59]. However, we note that there are some significant differences. First, there are no overlaps between particles below point $J$. At point $J$, the number of overlaps jumps discontinuously from 0 to $Z_{c}$ $=2 d$ (where $d$ is the dimensionality of space). In rigidity percolation, by contrast, the number of bonds increases continuously [60]. Second, in our case, the number of zero- 
frequency modes jumps discontinuously from $d N$ below point $J$ to $d\left(1+N_{r}\right)$ (which accounts for the translation of the entire system and the $N_{r}$ rattlers) above point $J$. This is again different from rigidity percolation, where the number of floppy modes decreases continuously [60]. Third, above point $J$, the spanning rigid cluster has a fractal dimension of $d$, the same as the space dimension, while in rigidity percolation, the spanning rigid cluster has a fractal dimension lower than $d$ [60]. Finally, the correlation length exponent that we extract from the finite-size scaling of the peak of the distribution of jamming thresholds, $\nu \approx 0.7$, is significantly lower than that calculated for rigidity percolation, $\nu \approx 1.2$ [60].

\section{IMPLICATIONS OF POINT $\boldsymbol{J}$ FOR THE GLASS TRANSITION}

We have shown that point $J$ marks a well-defined transition from the unjammed to the jammed state. Because the conjugate gradient method allows us to probe the infinitetime behavior of the system, we have been able to show that the system develops a truly static shear modulus at point $J$. Where point $J$ lies with respect to the jamming surface depicted in Fig. 1 depends on one's definition. Since the glass transition line is usually defined as the temperature where the relaxation reaches some large but finite threshold value, point $J$ in this definition strictly lies within the jammed phase since the relaxation time there is infinite. Since point $J$ lies just below the jamming surface of the phase diagram, one might expect it to control behavior in its vicinity if it is indeed a critical point. If so, it may be the long sought-after phase transition underlying the glass transition. In this section, we discuss why we suspect that the physics of point $J$ may hold clues for understanding the entire jamming surface of Fig. 1, including the glass transition itself.

One might wonder why point $J$ is important to real glassforming liquids, where there are not only finite-ranged repulsive interactions, such as those we have included in our calculations, but also longer-ranged attractions. The jamming phase diagram for a real liquid would look quite different from the one depicted in Fig. 1. In addition to the jamming surface, one has to consider the vapor-liquid phase coexistence curve since particles can attract one another. In Fig. 18, we have sketched the jamming phase diagram in the $T-1 / \phi$ plane when attractions are present. For simplicity, we have explicitly assumed that there is no possibility of crystallization. (If crystallization were taken into account, then the liquid that coexists with vapor could be metastable to the crystal.) In Fig. 18, the glass transition temperature decreases with increasing $1 / \phi$ and eventually crosses the liquid-vapor coexistence region at $\left(T_{x}, 1 / \phi_{x}\right)$, as shown. Once the glass transition curve crosses the left-hand side of the coexistence curve, which represents the lowest accessible liquid density, a variety of states can be obtained depending on the quench history. The dashed part of the glass transition curve, which ends at point $J$, is not necessarily accessible to systems with liquid-vapor phase transitions.

Even though point $J$ does not necessarily exist for real liquids, it can still influence the glass transition. In systems

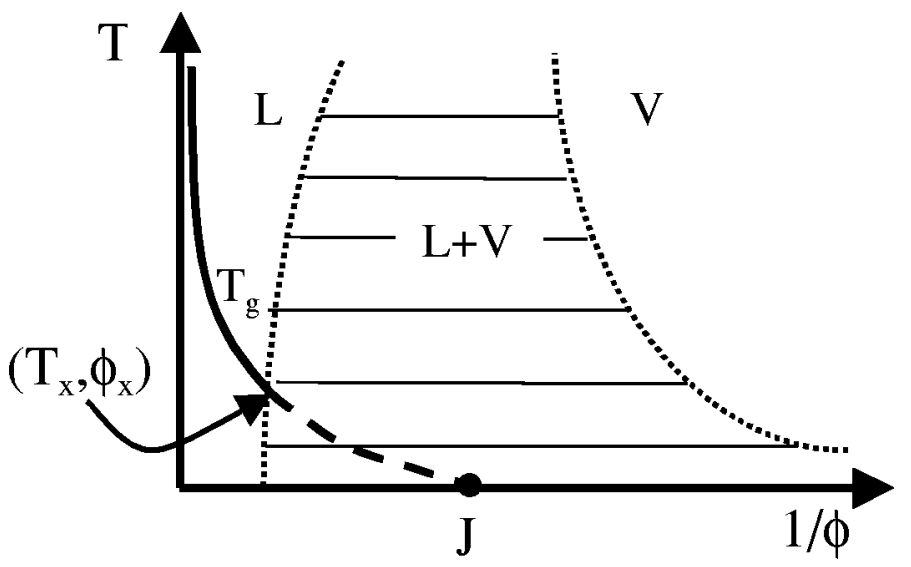

FIG. 18. A sketch of the jamming phase diagram in the $T-1 / \phi$ plane for a system with short-ranged repulsion and longer-ranged attraction. For simplicity, we have assumed that crystallization does not occur. The jammed region lies underneath the curve marked $T_{g}$. Beyond $\left(T_{x}, 1 / \phi_{x}\right)$, where the glass transition curve crosses the liquid density at coexistence, the available states depend on quench history.

with short-ranged repulsions and longer-ranged attractions, there is still a well-defined distance at which the repulsion vanishes; this is the position of the minimum in the pair potential. As with the theory of liquids, attractions are a small perturbation to the strong repulsive core; they merely hold the system at a sufficiently high density so that the repulsions can come into play [61]. We therefore expect the behavior we find near point $J$ to be a good approximation to the behavior of liquids down to the density at which the glass transition line crosses the liquid-vapor coexistence curve.

\section{A. Significance of divergence in pair correlation function}

We noted above that the first peak of the pair correlation function $g(r)$ diverges at point $J$. This has two consequences that have been observed in studies of the glass transition. The first has to do with the static structure factor $S(k)$, measured from scattering experiments, and the second has to do with the emergence of a peak in the distribution of normal forces, $P(F)$, as measured experimentally in granular [62] and colloidal [63] systems, and numerically in previous work on models of glass-forming liquids [10].

At point $J$, the first peak of $g(r)$ is infinitely high and narrow. This property elucidates one heretofore puzzling aspect of studies of supercooled liquids. The static structure factor $S(k)$ is related by a Fourier transform to $g(r)$ so the $\delta$-function peak in $g(r)$ produces oscillations in $S(k)$. There will not be a divergence in $S(k)$ at any wave vector $k$. This is different from what one finds at a critical point where there is a diverging susceptibility at some value of $k$. (In the case of a ferromagnetic transition, this would be the magnetic susceptibility at $k=0$.) Thus, the signature of the transition at point $J$ is different from that observed in ordinary secondorder phase transitions. As one moves away from point $J$ into the jammed region, the $\delta$ function in $g(r)$ broadens and decreases in height, but the oscillations in $S(k)$ persist. Representative plots of $S(k)$ at two different values of $\phi-\phi_{c}$ are 


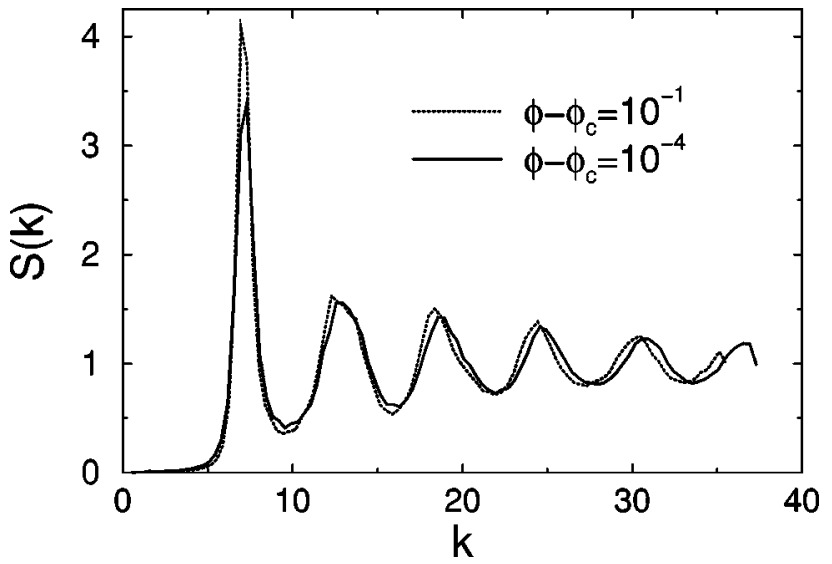

FIG. 19. Static structure factor $S(k)$ at $\phi-\phi_{c}=10^{-1}$ and $10^{-4}$ for a 3D monodisperse system with harmonic repulsions.

shown in Fig. 19. These are qualitatively similar to experimental results for $S(k)$ at high $k$, which also show oscillations [64]. This clarifies why searches for structural signatures of the glass transition that have examined the shape of $S(k)$, either at small $k$ or in the vicinity of the first peak, have not found a divergent behavior.

It has long been recognized that the first peak of $g(r)$ rises and sharpens as the temperature is lowered toward the glass transition. However, the change of behavior as one crosses the glass transition is only quantitative. A criterion suggested many years ago [65] that the glass transition occurs when the first peak reaches a threshold height, seems rather arbitrary. In a previous study [10], we showed that there is a qualitative change in a quantity closely related to $g(r)$. This is the distribution of normal forces $P(F)$ :

$$
P(F) d F \propto r^{d-1} g(r) d r,
$$

where $d$ is the dimensionality of the system. This quantity has been measured experimentally at the boundaries of static granular packings [62] and in the interior of colloidal glasses [63]. In all these studies of jammed systems, $P(F)$ was found to contain a peak. Our previous studies show that a peak develops in $P(F)$ as the jamming surface is approached by lowering $T$, increasing $\phi$, or decreasing $\Sigma[10,66]$. This signature was observed for all the potentials we have studied, including the full Lennard-Jones interaction, the WeeksChandler-Andersen (WCA) interaction [61], harmonic repulsions, and Hertzian repulsions. Thus, the development of a peak in $P(F)$ provides a signature of the onset of jamming from purely structural data. From Eq. (20), one can show that $P(F)$ develops a peak only if the first peak of $g(r)$ is sufficiently high and narrow. The criterion for a peak in $P(F)$ is

$$
\frac{d \ln g}{d r}=\frac{1-d}{r}+\frac{d^{2} F / d r^{2}}{d F / d r} .
$$

The fact that the onset of jamming is correlated with the first peak of $g(r)$ becoming high and narrow enough suggests that the entire jamming surface may be controlled by point $J$.

In order for a system to jam, it must be able to support shear stress for a very long time. The stress is supported

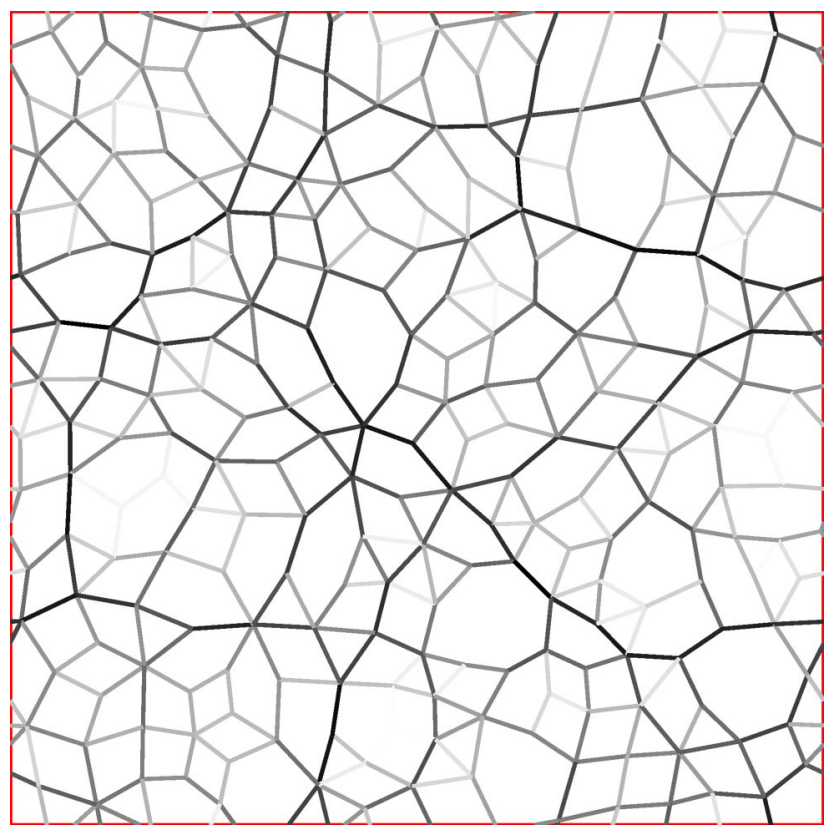

FIG. 20. Network of interparticle forces for a 2D bidisperse system with harmonic repulsions at $\phi-\phi_{c}=10^{-4.5}$ and $N=256$. The intensity of the line shading is proportional to the magnitude of the interparticle force.

through a network of interparticle forces, suggesting that an order parameter for jamming may be found in the nature of such a network. Forces on a particle must either be balanced by other forces or give rise to accelerations. At high temperatures, there is a lot of kinetic energy, and particles are constantly accelerated by unbalanced forces. At lower temperatures, however, the forces on particles tend to balance more because accelerations are smaller, and at zero temperature, forces on particles balance perfectly so that the system is mechanically stable at packing fractions above point $J$. The resulting network of forces at $T=0$ is shown just above the onset of jamming in Fig. 20. The order parameter for the glass transition presumably depends on at least a threeparticle quantity in order to characterize the force network. However, $P(F)$, which is only a two-particle quantity, clearly couples to the force network. A peak in $P(F)$ reflects the existence of the network because the forces on all particles can only balance if they are of roughly the same magnitude. This intuition highlights the importance of point $J$ to the glass transition. At $T=0$, as the packing fraction is increased through point $J$, the number of overlaps jumps from $Z=0$ (no force network) to $Z=2 d$ (a dense force network as shown in Fig. 20). Thus, point $J$ marks the development of a force network that supports shear stress.

\section{B. Significance of anomalous low-frequency modes in density of states}

Perhaps, the most striking evidence that the physics at point $J$ may be related to the nature of glasses and the glass transition is to be found in the behavior of the density of vibrational states at low frequencies. In contrast to our expectation that the density of states should vary as $D(\omega)$ 
$\propto \omega^{2}$ at low frequencies (in three dimensions), we find that at point $J$, the density of states approaches a nonzero constant value as $\omega \rightarrow 0$ (see Sec. II G).

We suspect that these extra low-frequency modes are primarily transverse in nature. It is clear from the behavior of the zero- and infinite-time shear moduli $G_{0}$ and $G_{\infty}$ that the transverse modes must become increasingly soft due to the relaxation allowed by non-affine deformations as $\phi$ approaches $\phi_{c}$. The ratio $G_{0} / G_{\infty}$ diverges at this point (see Sec. II H 1). The bulk modulus, in contrast, does not show any particular softening due to nonaffine relaxations and $B_{0} / B_{\infty}$, is a constant as $\phi$ approaches $\phi_{c}$ (see Sec. II H 1 ). This suggests that the anomalous low-frequency modes are more transverse than longitudinal in character. Moreover, since $G_{0} / G_{\infty}$ diverges, and the difference between $G_{0}$ and $G_{\infty}$ arises from spatially inhomogeneous nonaffine relaxations, we expect that there must be significant high-wave vector contributions mixed into the anomalous modes [67].

As $\phi \rightarrow \phi_{c}^{+}$, we also know that the normal modes are becoming more anharmonic. This was shown in Fig. 2 where it is clear that the linear region of the stress versus strain curves becomes smaller as $\phi_{c}$ is approached. The effect of this anharmonicity still needs to be determined.

Our results, that anomalous low-frequency vibrational modes proliferate and herald unjamming as $\phi$ approaches point $J$, are of clear relevance to a large body of experimental data on excess vibrational modes in glasses. Two results reflect these excess states rather directly. The first is the boson peak, measured by light and $x$-ray scattering [68] and in simulations [69], which indicates an excess of vibrational states at low frequencies, above those predicted by Debye behavior $\left[D(\omega) \propto \omega^{2}\right.$ in three dimensions]. The second is the low-temperature specific heat of glasses:

$$
c_{v}=A_{\text {Debye }} T^{3}+B T+C_{\text {excess }} T^{3} .
$$

In addition to the Debye term from long-wavelength sound modes, there is a linear term in the specific heat and an excess $T^{3}$ term above that predicted by the velocities of sound. The linear term has been ascribed to the existence of a new type of mode: two-level tunneling systems $[1,70]$. We note that a constant density of states as we have found at point $J$, would, by itself, produce a linear term without the necessity of assuming a new set of tunneling excitations. However, since glasses exist well above point $J$, we would not expect such a linear term to persist all the way down to zero temperature. Nevertheless, there is still a remarkable excess density of states even far away from point $J$ which would contribute to both the excess $T^{3}$ term and to the boson peak - both strong signatures of glassy behavior.

\section{CONCLUSIONS AND CONJECTURES}

We have demonstrated a number of remarkable properties of one special point on the jamming phase diagram that occurs at zero temperature and zero applied shear stress. We have shown that this point $J$ is the onset of jamming in both the bulk and shear modulus, it is well defined in the $N \rightarrow \infty$ limit and provides a clean definition of random close packing. In many ways, it behaves as a critical point, while in others it has properties not normally associated with a second-order phase transition. For example, many quantities, such as pressure and shear modulus, scale as power laws with $\phi-\phi_{c}$, but the scaling exponents depend on the interparticle potential and not on dimension.

From the finite-size scaling results that we have reported, one might also conjecture how quenched disorder imposed externally (such as from pinning sites in a flux lattice or from optical traps in a colloidal suspension) would affect the nature of the jamming phase diagram. If we assume that the spacing between defects limits the correlation length in the system, instead of the finite size of the box that we employed in these studies, then we would expect that the jamming threshold at point $J$ would be smeared out in much the same way as we find in finite-sized systems. Thus, if we were to add a "quenched disorder" axis to the jamming phase diagram, one of the implications of our work would be that as more quenched disorder is added, the distribution in jamming thresholds will broaden.

Our studies here have been confined to purely frictionless particles. We suspect that for systems with frictional interactions, the distribution of jamming thresholds should broaden as well. This would be in accord with experimental observations that static frictional packings can exist over a wide range of densities.

Perhaps, most significant is that at point $J$ many of the properties of disordered glassy systems have their most pronounced expression. Just as a crystal is the most ordered of states, point $J$ may be considered to be the most disordered of states. As at a critical point, where the correlations across the entire system are most easily observable, at point $J$ the nature of the disordered phase is most plainly seen. The constant density of low-frequency normal modes and the divergence in the first peak in $g(r)$ are two extraordinary examples. Both are sensitive to global properties of the system; $D(\omega)$ because it deals with the longest wavelength modes in the system and $g(r)$ because the overlap between all particles simultaneously goes to zero. In addition, both of these observations have implications for how real glassy systems behave. It is tempting to think that point $J$ may provide a key to understanding the nature of the entire surface in the jamming phase diagram and to argue that the properties of other glassy states should be understood as a perturbation around this "most disordered" of states. Thus, one might say that point $J$ represents the epitome of disorder and the essence of glassiness.

\section{ACKNOWLEDGMENTS}

We thank Bulbul Chakraborty, Sue Coppersmith, Morrell Cohen, Doug Durian, Gary Grest, Leo Kadanoff, Steve Langer, Dov Levine, Gilles Tarjus, Denis Weaire, and Tom Witten for helpful discussions. We also gratefully acknowledge the support of Grant Nos. NSF-DMR-0087349 (A.J.L.) and NSF-DMR-0089081 (S.R.N.). 
[1] P.W. Anderson, Science 267, 1615 (1995).

[2] L.P. Kadanoff, Rev. Mod. Phys. 71, 435 (1999).

[3] P.G. de Gennes, Rev. Mod. Phys. 71, S374 (1999).

[4] See Jamming and Rheology, edited by A.J. Liu and S.R. Nagel (Taylor \& Francis, New York, 2001), and references therein.

[5] H.M. Jaeger, S.R. Nagel, and R.P. Behringer, Rev. Mod. Phys. 68, 1259 (1996).

[6] P.N. Pusey, in Liquids, Freezing and the Glass Transition, Part II, Les Houches Summer School Proceedings Vol. 51, edited by J.-P. Hansen, D. Levesque, and J. Zinn-Justin (Elsevier, Amsterdam, 1991), Chap. 10.

[7] D.J. Durian and D.A. Weitz, "Foams," in Kirk-Othmer Encyclopedia of Chemical Technology, 4th ed., edited by J.I. Kroschwitz (Wiley, New York, 1994), Vol. 11, p. 783.

[8] M.D. Ediger, C.A. Angell, and S.R. Nagel, J. Phys. Chem. 100, 13200 (1996); P.G. Debenedetti and F.H. Stillinger, Nature (London) 410, 259 (2001).

[9] G. Tarjus, D. Kivelson, and P. Viot, J. Phys.: Condens. Matter 12, 6497 (2000); K. Dixon, L. Wu, S.R. Nagel, B.D. Williams, and J.P. Carini, Phys. Rev. Lett. 65, 1108 (1990).

[10] C.S. O'Hern, S.A. Langer, A.J. Liu, and S.R. Nagel, Phys. Rev. Lett. 86, 111 (2001).

[11] D. Kivelson and G. Tarjus, J. Non-Cryst. Solids 235-237, 86 (1998); G. D’Anna and G. Gremaud, Nature (London) 413, 407 (2001); P.N. Pusey and W. van Megan, Phys. Rev. Lett. 59, 2083 (1987).

[12] W. Kob, C. Donati, S.J. Plimpton, P.H. Poole, and S.C. Glotzer, Phys. Rev. Lett. 79, 2827 (1997); B. Doliwa and A. Heuer, ibid. 80, 4915 (1998); M.D. Ediger, Annu. Rev. Phys. Chem. 51, 99 (2000); E.R. Weeks, J.C. Crocker, A.C. Levitt, A. Schofield, and D.A. Weitz, Science 287, 627 (2000); S.A. Langer and A.J. Liu, J. Phys. Chem. B 101, 8667 (1997).

[13] A.J. Liu and S.R. Nagel, Nature (London) 396, 21 (1998).

[14] L.F. Cugliandolo, J. Kurchan, and L. Peliti, Phys. Rev. E 55, 3898 (1997).

[15] L. Berthier and J.-L. Barrat, Phys. Rev. Lett. 89, 095702 (2002); J. Chem. Phys. 116, 6228 (2002).

[16] H.A. Makse and J. Kurchan, Nature (London) 415, 614 (2002).

[17] I.K. Ono, C.S. O'Hern, D.J. Durian, S.A. Langer, A.J. Liu, and S.R. Nagel, Phys. Rev. Lett. 89, 095703 (2002).

[18] S.A. Langer and A.J. Liu, Europhys. Lett. 49, 68 (2000).

[19] C.S. O'Hern, A.J. Liu, and S.R. Nagel (unpublished).

[20] C. Alba-Simionesco, D. Kivelson, and G. Tarjus, J. Chem. Phys. 116, 5033 (2002).

[21] T.G. Mason, M.-D. Lacasse, G.S. Grest, D. Levine, J. Bibette, and D.A. Weitz, Phys. Rev. E 56, 3150 (1997).

[22] A. Saint-Jalmes and D.J. Durian, J. Rheology 43, 1411 (1999).

[23] W. Götze and L. Sjogren, Phys. Rev. A 43, 5442 (1991).

[24] M. Fuchs and M.E. Cates, Phys. Rev. Lett. 89, 248304 (2002).

[25] L. Berthier, J.-L. Barrat, and J. Kurchan, Phys. Rev. E 61, 5464 (2000).

[26] V. Trappe, V. Prasad, L. Cipelletti, P.N. Segre, and D.A. Weitz, Nature (London) 411, 772 (2001).

[27] C.S. O'Hern, S.A. Langer, A.J. Liu, and S.R. Nagel, Phys. Rev. Lett. 88, 075507 (2002).

[28] K.L. Johnson, Contact Mechanics (Cambridge University Press, Cambridge, 1985).

[29] D.N. Perera and P. Harrowell, Phys. Rev. E 59, 5721 (1999).

[30] R.J. Speedy, J. Chem. Phys. 110, 4559 (1999).
[31] W.H. Press, B.P. Flannery, S.A. Teukolsky, and W.T. Vetterling, Numerical Recipes in Fortran 77 (Cambridge University Press, New York, 1986).

[32] F.H. Stillinger and T.A. Weber, Science 225, 983 (1984); Phys. Rev. A 28, 2408 (1983).

[33] M.P. Allen and D.J. Tildesley, Computer Simulation of Liquids (Oxford University Press, New York, 1987).

[34] M. Born and K. Huang, Dynamical Theory of Crystal Lattices (Clarendon Press, Oxford, 1988).

[35] G.Y. Onoda and E.G. Liniger, Phys. Rev. Lett. 64, 2727 (1990).

[36] R.J. Speedy, J. Phys.: Condens. Matter 10, 4185 (1998).

[37] J.G. Berryman, Phys. Rev. A 27, 1053 (1983).

[38] S. Torquato, T.M. Truskett, and P.G. Debenedetti, Phys. Rev. Lett. 84, 2064 (2000).

[39] A.R. Kansal, S. Torquato, and F.H. Stillinger, Phys. Rev. E 66, 041109 (2002).

[40] B.D. Lubachevsky and F.H. Stillinger, J. Stat. Phys. 60, 561 (1990).

[41] S. Alexander, Phys. Rep. 296, 65 (1998).

[42] L.E. Silbert, D. Ertas, G.S. Grest, T.C. Halsey, and D. Levine, Phys. Rev. E 65, 031304 (2002).

[43] M.D. Rintoul and S. Torquato, J. Chem. Phys. 105, 9258 (1996).

[44] R.J. Bell, in Vibration Properties of Solids, edited by G. Gilat, Methods in Computational Physics: Advances in Research and Applications Vol. 15 (Academic, New York, 1976), p. 216.

[45] A. Rahman, M.J. Mandell, and J.P. McTague, J. Chem. Phys. 64, 1564 (1976); J.P. McTague, M.J. Mandell, and A. Rahman, ibid. 68, 1876 (1978).

[46] S.R. Nagel, G.S. Grest, S. Feng, and L.M. Schwarz, Phys. Rev. B 34, 8667 (1986).

[47] S. John, H. Sompolinsky, and M.J. Stephen, Phys. Rev. B 27, 5592 (1983).

[48] G. Seeley and T. Keyes, J. Chem. Phys. 91, 5581 (1989).

[49] H.R. Schober and B.B. Laird, Phys. Rev. B 44, 6746 (1991).

[50] A. Tanguy, J.P. Wittmer, F. Leonforte, and J.-L. Barrat, Phys. Rev. B 66, 174205 (2002).

[51] D.J. Lacks, J. Chem. Phys. 111, 9137 (1999).

[52] S. Sastry, Nature (London) 409, 164 (2001).

[53] D.J. Durian, Phys. Rev. Lett. 75, 4780 (1995); Phys. Rev. E 55, 1739 (1997).

[54] H.A. Makse, D.L. Johnson, and L.M. Schwartz, Phys. Rev. Lett. 84, 4160 (2000).

[55] E. Clément (private communication).

[56] E.R. Weeks et al. (to be published).

[57] M.B. Hastings, C.J. Oslon Reichardt, and C. Reichardt, Phys. Rev. Lett. 90, 098302 (2003).

[58] J.V. Evans, Rev. Mod. Phys. 65, 1281 (1993).

[59] F. Bolton and D. Weaire, Phys. Rev. Lett. 65, 3449 (1990).

[60] D.J. Jacobs and M.F. Thorpe, Phys. Rev. E 53, 3682 (1996); C. Moukarzel and P.M. Duxbury, ibid. 59, 2614 (1999).

[61] J.D. Weeks, D. Chandler, and H.C. Anderson, J. Chem. Phys. 54, 5237 (1971).

[62] J.M. Erikson, N.W. Mueggenburg, H.M. Jaeger, and S.R. Nagel, Phys. Rev. E 66, 040301 (2002); D.L. Blair, N.W. Mueggenburg, A.H. Marshall, H.M. Jaeger, and S.R. Nagel, ibid. 
63, 041304 (2001); D.M. Mueth, H.M. Jaeger, and S.R. Nagel, ibid. 57, 3164 (1998).

[63] J. Brujic, S.F. Edwards, D.V. Grinev, I. Hopkinson, D. Brujic, and H.A. Makse, Faraday Discuss. 123, 207 (2003).

[64] S.C. Moss and D.L. Price, in Physics of Disordered Materials, edited by D. Adler, H. Fritzche, and S.R. Ovshinsky (Plenum Press, New York, 1985).

[65] H.R. Wendt and F.F. Abraham, Phys. Rev. Lett. 41, 1244 (1978).

[66] L.E. Silbert, D. Ertaş, G.S. Grest, T.C. Halsey, and D. Levine, Phys. Rev. E 65, 051307 (2002).
[67] S.R. Nagel, G.S. Grest, and A. Rahman, Phys. Rev. Lett. 53, 368 (1984).

[68] C. Masciovecchio, G. Ruocco, F. Settem, M. Krisch, R. Verbeni, U. Bergmann, and M. Soltwisch, Phys. Rev. Lett. 76, 3356 (1996); S. Kojima and M. Kodama, Physica B 263-264, 336 (1999).

[69] G. Parisi, Nature (London) 422, 289 (2003); V.L. Gurevich, D.A. Parshin, and H.R. Schober, Phys. Rev. B 67, 094203 (2003).

[70] Amorphous Solids: Low Temperature Properties, edited by W.A. Phillips (Springer-Verlag, Berlin, 1981). 